\title{
Evaluation of the first GOCE static gravity field models using terrestrial gravity, vertical deflections and EGM2008 quasigeoid heights
}

\author{
C. Hirt \\ Western Australian Centre for Geodesy \& The Institute for Geoscience Research, \\ Curtin University of Technology, GPO Box U1987, Perth, WA 6845, Australia \\ Email: c.hirt@curtin.edu.au
}

\section{T. Gruber}

Institute of Astronomical and Physical Geodesy, Technische Universität München, Arcisstrasse 21, 80333 München, Germany

Email: Thomas.Gruber@tum.de

\author{
W.E. Featherstone \\ Western Australian Centre for Geodesy \& The Institute for Geoscience Research, \\ Curtin University of Technology, GPO Box U1987, Perth, WA 6845, Australia \\ Email: w.featherstone@curtin.edu.au
}

\begin{abstract}
Recently, four global geopotential models (GGMs) were computed and released based on the first two months of data collected by the GOCE (Gravity field and steady-state Ocean Circulation Explorer) dedicated satellite gravity field mission. Given that GOCE is a technologically complex mission and different processing strategies were applied to real space-collected GOCE data for the first time, evaluation of the new models is an important aspect. As a first assessment strategy, we use terrestrial gravity data over Switzerland and Australia and astrogeodetic vertical deflections over Europe and Australia as ground-truth data sets for GOCE model evaluation. We apply a spectral enhancement method (SEM) to the truncated GOCE GGMs to make their spectral content more comparable with the terrestrial data. The SEM utilises the high-degree bands of EGM2008 and residual terrain model (RTM) data as a data source to widely bridge the spectral gap between the satellite and terrestrial data. Analysis of RMS (root mean square) errors is carried out as a function of (i) the GOCE GGM expansion degree and (ii) the four different GOCE GGMs. The RMS curves are also compared against those from EGM2008 and GRACE-based GGMs. As a second assessment strategy, we compare global grids of GOCE GGM and EGM2008 quasigeoid heights. In connection with EGM2008 error estimates, this allows location of regions where GOCE is likely to deliver improved knowledge on the Earth's gravity field. Our ground truth data sets, together with the EGM2008 quasigeoid comparisons, signal clear improvements in the spectral band $\sim 160-165$ to $\sim 180-185$ in terms of spherical harmonic degrees for the GOCE-based GGMs, fairly independently of the individual GOCE model used. The results from both assessments together provide strong evidence that the first two months of GOCE observations improve the knowledge of the Earth's static gravity field at spatial scales between $\sim 125$ and $\sim 110 \mathrm{~km}$, particularly over parts of Asia, Africa, South America and Antarctica, in comparison to the pre-GOCE-era.
\end{abstract}


Keywords: GOCE, validation, spectral enhancement, gravity, vertical deflections, EGM2008, GRACE

\section{Introduction}

ESA's (the European Space Agency's) GOCE (Gravity field and steady-state Ocean Circulation Explorer) is the first-ever satellite mission that deploys dedicated gradiometry in space to produce a homogeneous, highly-accurate, near-global map of the Earth's static gravity field (e.g., Rummel et al. 2002, Visser et al. 2002; Drinkwater et al. 2003). The main objective of the GOCE mission is to deliver a gravity field model at the $\sim 1-2 \mathrm{~cm}$ accuracy level for geoid undulations and at the 1-2 mGal level for gravity anomalies (Rummel 2005) down to a target resolution of $\sim 100 \mathrm{~km}$, which equates to degree 200 in terms of spherical harmonics. GOCE utilises space-borne gravity gradiometry in combination with GPS-based (Global Positioning System) high-low SST (satellite-to-satellite tracking) e.g., Rummel et al. (2002), Gruber (2008), Rummel et al. (2009).

Launched on 17 March 2009, GOCE is currently sensing the Earth's gravity field, and is expected to collect data until 2012. GOCE is complementary to previous satellite gravity field missions, such as GRACE (Gravity Recovery And Climate Experiment; e.g., Reigber et al. 2005, Tapley et al. 2005), which significantly improved the knowledge of the low- to medium-frequency constituents of the Earth's static gravity field. Because of the complementary character of the missions, GOCE augmented by GRACE in the lower frequencies is expected to provide an even better picture of Earth's global gravity field than GOCE alone (Pail et al. 2010a).

Based on the first two months of GOCE observations, four GOCE-based global geopotential models (GGMs) were recently made available to the public via ESA (http://www.esa.int) and the IAG's (International Association of Geodesy's) ICGEM (International Centre for Global Earth Models, http://icgem.gfz-potsdam.de/ICGEM/) operated by GFZ German Research Centre for Geosciences Potsdam. The four new models (Table 1) differ in their maximum spherical harmonic degree and computation method (i.e., the way GOCE observations were modelled and processed to yield the GGM coefficients). As a further difference, auxiliary data sets (e.g., information from GRACE GGMs) were used as augmentation to some of the models (described later). This poses the question of the relative performance of the new GGMs: how closely do they approximate Earth's global gravity field and in which spectral bands do they deliver improved information?

Comparisons with independent gravity field functionals (as 'ground-truth') can be used to evaluate the performance of the new GOCE-based GGMs. Independent sources of gravity field information are, for instance, geoid undulations from GPS/levelling, gravity anomalies and astrogeodetic vertical deflections. The main problem with such comparisons is the different spectral content of the GGMs and terrestrial observations (e.g., Gruber 2009; Ihde et al. 2010). Because the spatial resolution of GGMs is limited by their maximum degree, there is always an omission error (i.e., neglect of high-degree spectral content) in the GGM-derived functionals. In contrast, terrestrial observations contain the full spectral signal.

Therefore, GGM evaluation usually requires the data sets involved (GGM functionals and terrestrial observations) to be comparable in terms of spectral content. There are (at least) two different strategies to solve this problem. 
1. Low-pass filtering of the terrestrial data to remove their high-frequency constituents (e.g., Gruber 2004; Ihde et al. 2010).

2. Spectral enhancement of the GGM-based functionals in the high-and very-highfrequency range to become more comparable with the full-spectrum terrestrial observations (Pavlis et al. 2007; Gruber 2009; Hirt 2010; Hirt et al. 2010a; Gruber et al. 2011). For the spectral enhancement, the high-frequency bands of EGM2008 (Pavlis et al. 2008) and residual terrain model (RTM) data (e.g., Forsberg 1984; Hirt 2010) can be utilised.

The application of the spectral enhancement method (SEM) for GOCE GGM validation with scattered ground-truth data is one subject of this paper. Low-pass filtering using methods such as spectral filters, Gaussian filters or least-squares collocation (LSC) may perform well when the terrestrial data is available in some suitable arrangement, for instance, profiles (e.g., Ihde et al. 2010), grids, or at densely scattered locations. However, low-pass filtering is a delicate task in the case of very irregularly or scarcely distributed stations, if possible at all. In contrast, the SEM (Sect. 2) can be used for GGM validation independent of the spatial distribution of the terrestrial data. This is an important advantage when using irregularly distributed ground truth observations as is often the case with GPS/levelling, terrestrial gravimetry or astrogeodetic vertical deflections.

The present paper uses two complementary assessment techniques to evaluate the new GOCE-based GGMs. The first uses the SEM along with scattered terrestrial gravity over Switzerland and Australia and vertical deflection data over Europe and Australia (Sects. 2 to 4). The second compares global grids of EGM2008 and GOCE quasigeoid heights. Together with EGM2008 error estimates, world-wide insight into GOCE's performance is gained (Sect. 5).

The first assessment technique utilises observed ground-truth gravity and vertical deflections. Gravity observations (first radial derivatives of the disturbing potential) and vertical deflections (first horizontal derivatives of the disturbing potential) possess significant spectral power in the medium and high frequencies (Schwarz 1985, Jekeli 1999, Torge 2001, p. 274), so are useful complements to GGM evaluation using GPS/levelling points (Gruber 2009; Ihde et al. 2010; Gruber et al. 2011). The two vertical deflection data sets are particularly valuable, as they are fully independent of any GGM used in this paper. The deflections can be used to investigate in which spectral windows the GOCE-based models provide new information on the Earth's gravity field in our test areas.

In addition to the four GOCE-based GGMs, we also include two GRACE-only GGMs and EGM2008 (Sect. 3), so as to identify improvements of the current knowledge of the Earth's gravity field coming from the new GOCE-based models. Analysis of the RMS (root mean square) residuals between the terrestrial data sets and spectrally enhanced GGM functionals (Sect. 4) indicates the relative performance of all GGMs involved, and identifies the actual spectral resolution of the models, which is the degree of signal loss (see Gruber et al. 2011); also see Sect. 4.

The second assessment technique is based on global comparisons between bandlimited EGM2008 and GOCE GGM quasigeoid heights (Sect. 5) and supplements the groundtruth comparisons of Sect. 4. When using identical spectral bands, this evaluation method does not require any modelling of omitted signals, thus avoiding the problem of different 
spectral content. Along with background knowledge of the global structure of EGM2008 commission errors (i.e., uncertainties in the GGM), the EGM2008 vs. GOCE comparisons indicate regions and continents where the GOCE models are likely to deliver improved gravity field data (Sect. 5).

Prior to describing the SEM and GOCE GGMs, it is useful to define the terms long, medium, short and very-short wavelength as used in this study to address the different spectral constituents of Earth's gravity field (see also Torge 2001, p. 74). The term long denotes here the spectral band of spherical harmonic degree $2 \ldots 100$ (wavelengths longer than $\sim 400 \mathrm{~km}$ ). Medium wavelengths range from $\sim 400 \mathrm{~km}$ to $\sim 200 \mathrm{~km}$ (spherical harmonic degrees of $\sim 100$ to about $\sim 200$, or a resolution down to $\sim 100 \mathrm{~km}$, which is the target resolution of GOCE). Short wavelengths cover the spherical harmonic degrees of 200 to 2190 (wavelengths between $\sim 200 \mathrm{~km}$ and $\sim 18 \mathrm{~km}$, or a spatial resolution between $100 \mathrm{~km}$ and $9 \mathrm{~km}$ ). Finally, very-short denotes those features beyond the resolution of EGM2008, i.e., wavelengths less than 18 $\mathrm{km}$. It is acknowledged that other definitions exist in the geodetic literature, however, the terminology here is tailored to the GOCE validation with SEM.

\section{The Spectral Enhancement Method}

\subsection{Principle}

The basic idea of the SEM is to source relevant auxiliary data to bridge the spectral gap of the GOCE-based GGM functionals as far as possible, so that the terrestrial observations are better approximated and the omission error does not obscure the comparisons. In the SEM, the spectral gap between the GOCE-GGMs and the terrestrial observations is bridged partially by a combination of: (i) the high-degree spectral bands of EGM2008, and (ii) omission error estimates sourced from RTM data, providing information on the very-short wavelength gravity field constituents. Of course, there remains an omission error due to the limited RTM resolution.

The SEM strategy has been applied in Gruber et al. (2011) for the validation of GOCE-GGMs at GPS/levelling points, while Hirt et al. (2010a) used RTM omission error estimates (Hirt 2010) together with observed astrogeodetic vertical deflections to assess EGM2008. Pavlis et al. (2007) used SEM in the development of EGM2008 over areas where proprietary gravity anomaly data were only available, and the use of these data was only permitted to a spatial resolution of 15'x15'. Pavlis et al. (2007) used the RTM estimates to augment the spectral content of these anomalies from degree 721 to degree 2159 (in ellipsoidal harmonics). 

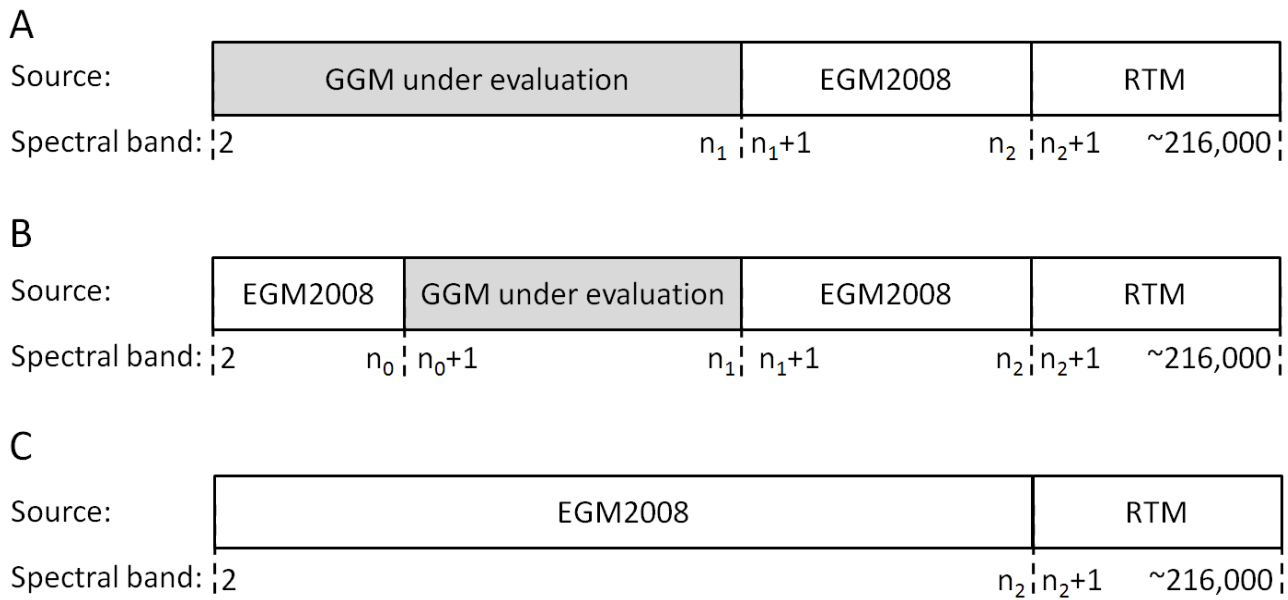

Fig. 1 Spectral enhancement principle for GGM evaluation. A (special case): GGM under evaluation substitutes EGM2008 spectral band $2 \ldots \mathrm{n}_{1}$ (spectral enhancement from $\mathrm{n}_{1}+1$ ...216,000). B (general case): GGM under evaluation substitutes EGM2008 spectral window $\mathrm{n}_{0}+1 \ldots \mathrm{n}_{1}$ (additional spectral enhancement in band 2... $\mathrm{n}_{0}$ ). C: EGM2008/RTM only solution (cf. Hirt et al. 2010a).

The SEM principle is exemplified in Fig. 1. The GOCE GGM under evaluation is expanded to spherical harmonic degree $\mathrm{n}_{1}$ (e.g., 200), with EGM2008 used to recover the spectral band $n_{1}+1$ to $n_{2}$ (e.g., 2,190). Beyond degree $n_{2}$, we use RTM omission error estimates to 'complete' the spectral content of the model as far as possible (e.g., 216,000) with current data sets (Fig 1A).

The strategy to investigate a specific spectral band $\left(n_{0}+1\right.$ to $\left.n_{1}\right)$ of the GOCE GGM is visualised in Fig 1B. Here, EGM2008 is not only used from $n_{1}+1$ to $n_{2}$, but also in spectral band 2 to $n_{0}$, with the GOCE GGM evaluated from $n_{0}+1$ to $n_{1}$ (e.g., 150 to 200). In other words, the GOCE-GGM substitutes EGM2008 within spectral window $n_{0}+1$ to $n_{1}$. Varying the combination degrees $n_{0}$ and $n_{1}$, as well as the GOCE-GGM, the RMS of the differences 'terrestrial observation minus GGM' can be used to indicate the performance of the different GGMs in any spectral window chosen.

Comparing against EGM2008/RTM 'benchmark' solutions (Fig. 1C) is another strategy to identify in which spectral bands the GOCE-based models deliver improved information on the Earth's static gravity field. However, this requires ground-truth data that are independent of all GGMs involved, which only holds for our vertical deflections (e.g., the gravity anomalies here were also used by the EGM2008 Development Team). The present study applies the SEM variants shown in Figs. 1A and 1C (also see Sect. 4).

\subsection{RTM omission error estimates}

To compute RTM omission error estimates, we construct the RTM (Forsberg 1984) as difference between the 3 arc second SRTM (Shuttle Radar Topography Mission) elevation model (ver4.1 by Jarvis et al. 2008) and the spherical harmonic expansion of the DTM2006.0 elevation data base (Pavlis et al. 2007), expanded to degree 2,160. Subtraction of DTM2006.0 spherical harmonic elevations from the SRTM elevations removes a large part of the 'spectral information' already implied by EGM2008 (expanded to degree 2,190), see Hirt 
(2010) and Hirt et al. (2010a,b) for a fuller description. The parallel use of EGM2008 to degree $\mathrm{n}_{2}=2,190$ and DTM2006.0 to degree 2,160 is recommended by EGM Development Team (Pavlis et al. 2008), and allows modelling of the EGM2008 omission error to a large extent in medium-elevated and rugged terrain (Hirt et al. 2010a, Hirt et al. 2010b).

The SRTM-DTM2006.0 residual topography possesses spectral energy beyond the resolution of EGM2008 (in spectral band $n_{2}+1=2,191$ to $~ 216,000$, which is equivalent to the SRTM resolution of 3 arc seconds). The RTM elevation grid is then used for forwardmodelling of the gravity field functionals (here vertical deflections and gravity disturbances), as implied by the residual topography [see Forsberg (1984); Nagy et al. (2000) and Hirt et al. (2010a) for the equations]. Our forward modelling is based on a uniform mass-density (2670 $\mathrm{kg} / \mathrm{m}^{3}$ ) for the residual topography, which is why any density anomalies at scales beyond the EGM2008 resolution are not modelled by the RTM approach (akin to a commission error of the RTM omission error estimates).

\subsection{Computation scheme}

The SEM can be applied for GGM evaluation with terrestrial gravity disturbances and vertical deflections using the following computation scheme. Naturally, it can also be applied to quasi/geoid heights from GPS/levelling (Gruber 2009; Hirt et al. 2010b; Gruber et al. 2011) or other 'ground-truth' data sources (e.g., torsion balance measurements or airborne gravimetry and airborne gravity gradiometry). The equations for the standard operations (e.g., spherical harmonic synthesis, forward modelling of terrain effects) are sufficiently and frequently detailed in the literature (e.g., Torge 2001; Forsberg 1984; Nagy et al. 2000; Hirt 2010), so are not duplicated here. For gravity observations, the SEM scheme reads:

1. Computation of gravity disturbances by subtracting the normal gravity at the ellipsoidal height of the station (Torge 2001 p. 117) from the observed gravity.

2. Synthesis of GGM gravity disturbances at the 3D coordinates of our gravity stations (geodetic latitude, longitude and ellipsoidal height), e.g., Torge (2001, p. 271). This is done by using the scattered point option of the harmonic_synth.f software (Holmes and Pavlis 2008). The synthesis computations are carried out separately for each GGM involved, and for a range of spectral windows from 2 to $\mathrm{n}_{1} \in[2,3, \ldots$, n_max_model]. EGM2008 is included here so as to allow computation of 'EGM2008only’ solutions.

3. Computation of EGM2008 gravity disturbances by repeating step 2 with spectral windows $\mathrm{n}_{1}+1$ to 2,190 .

4. Addition of the gravity disturbances from step 2 (spectral band 2 to $n_{1}$ ) and the complementary EGM2008 disturbances (band $n_{1}+1$ to 2,190) from step 3 . This gives a merger of gravity disturbances with spectral power in bands 2 to 2,190.

5. Optional: Construction of the GOCE GGM contributions in arbitrary spectral bands $\mathrm{n}_{0}+1$ to $\mathrm{n}_{1}$ (e.g., 120 to 240). For instance, this is achieved by adding a 'correction' EGM2008 (band 2 to $\mathrm{n}_{0}$ ) minus GOCE GGM (band 2 to $\mathrm{n}_{0}$ ) to the solutions of step 4.

6. Computation of the RTM gravity disturbances (spectral band 2,191 and well beyond). For each of the terrestrial stations, the required elevation grids (3 arc second SRTM and DTM2006.0) are extracted from our elevation data base (with the terrestrial station as centre and $\sim 50 \mathrm{~km}$ grid radius). The RTM elevations are used for mass- 
density forward modelling using the Curtin RTM software (Hirt et al. 2010a) based on the TC program (Forsberg 1984). For stations located 'within the residual topography', the [RTM] harmonic correction is applied, see also Forsberg and Tscherning (1981, p 7846) and Forsberg (1984, p. 40).

7. The sum of the RTM gravity disturbances (band 2191 and beyond) and synthesised gravity disturbances (band 2 to 2,190) from steps 4 and 5 gives our spectrally enhanced model gravity disturbances. They possess almost full spectral power and can be compared with the terrestrial observations.

8. RMS values are computed from the gravity disturbance differences 'observation minus model' (these are the results from step 1 minus results from step 7) and analysed as a function of (i) the GOCE GGM under evaluation and (ii) spectral band 2 to $\mathrm{n}_{1}$ (or $\mathrm{n}_{0}+1$ to $\mathrm{n}_{1}$ ) used.

For vertical deflections, the scheme is very similar. However, step 1 is not necessary and the harmonic correction (Forsberg 1984, p.40) of step 6, does not need to be applied. Additionally, two small corrections are applied to the GGM vertical deflections, converting them from Molodensky's definition in spherical approximation to Helmert vertical deflections in ellipsoidal approximation [see Jekeli (1999) and Hirt et al. (2010a) for the equations and further explanations].

\section{Data sets}

\subsection{GGMs}

This study evaluates the first four GOCE-based GGMs which were made available in 2010 via ICGEM. In addition, we include two GRACE-only GGMs and EGM2008 in our analyses (Table 1) so as to identify any improvements of the new GOCE-models over GGMs from the pre-GOCE-era. Common to the four GOCE-based models is that the first two months of GOCE observations (collected between November 2009 and January 2010) were used as input data. However, they differ in the processing strategies applied, the spectral resolution (maximum degree of expansion) and, importantly, by the type and amount of a-priori information ('background models') used in the model construction.

An overview of the computation methods used for GOCE-based gravity field recovery is given in Rummel et al. (2004). Detailed descriptions of the underlying computation methods will become available in the near future (e.g., Pail et al. 2011). Table 1 lists all GGMs used in this study, including the ESA and ICGEM product names of the GOCE models where applicable (note that ESA and ICGEM use different names). For the sake of legibility, we abbreviate the ESA product names as shown in Table 1.

Table 1 Overview of the GGMs used in this study. n_max_model is the maximum spherical harmonic degree of the model, $\mathrm{G}=$ terrestrial gravity, $\mathrm{A}=$ satellite altimetry.

\begin{tabular}{|l|l|l|l|}
\hline GGM & $\begin{array}{l}\text { Model } \\
\text { resolution } \\
\text { n_max_model }\end{array}$ & $\begin{array}{l}\text { Data } \\
\text { sources }\end{array}$ & $\begin{array}{l}\text { Method and } \\
\text { References }\end{array}$ \\
\hline GOCO01S & 224 & $\begin{array}{l}\text { GOCE, } \\
\text { GRACE }\end{array}$ & $\begin{array}{l}\text { Based on the time-wise } \\
\text { approach and combined } \\
\text { with GRACE }\end{array}$ \\
\hline
\end{tabular}




\begin{tabular}{|c|c|c|c|}
\hline & & & Pail et al. (2010a) \\
\hline $\begin{array}{l}\text { GOCE-DIR } \\
\text { (ESA product name: } \\
\text { EGM_GOC_2__20091101T000000_20100 } \\
\text { 110T235959_0002; } \\
\text { ICGEM product name: } \\
\text { GO_CONS_GCF_2_DIR) }\end{array}$ & 240 & $\begin{array}{l}\text { GOCE, } \\
\text { EIGEN- } \\
51 C \\
\text { (GRACE, } \\
\text { CHAMP,G, } \\
\text { A) }\end{array}$ & $\begin{array}{l}\text { Based on the direct } \\
\text { approach } \\
\text { Bruinsma et al. (2010), } \\
\text { Pail et al. (2011) }\end{array}$ \\
\hline $\begin{array}{l}\text { GOCE-TIM } \\
\text { (ESA product name: } \\
\text { EGM_GOC_2_20091101T000000_20100 } \\
\text { 111T000000_0002; } \\
\text { ICGEM product name: } \\
\text { GO_CONS_GCF_2_TIM) }\end{array}$ & 224 & GOCE & $\begin{array}{l}\text { Based on the time-wise } \\
\text { approach } \\
\text { Pail et al. (2010b), } \\
\text { Pail et al. (2011) }\end{array}$ \\
\hline $\begin{array}{l}\text { GOCE-SPW } \\
\text { (ESA product name: } \\
\text { EGM_GOC_2_20091030T005757_20100 } \\
\text { 111T073815_0002; } \\
\text { ICGEM product name: } \\
\text { GO_CONS_GCF_2_SPW) }\end{array}$ & 210 & $\begin{array}{l}\text { GOCE, } \\
\text { EGM2008 }\end{array}$ & $\begin{array}{l}\text { Based on the space-wise } \\
\text { approach } \\
\text { Miggliaccio et al. } \\
\text { (2010), } \\
\text { Pail et al. (2011) }\end{array}$ \\
\hline GGM03s & 180 & GRACE & $\begin{array}{l}\text { Based on GRACE inter- } \\
\text { satellite ranging and } \\
\text { SST } \\
\text { Tapley et al. (2007) }\end{array}$ \\
\hline ITG-GRACE2010s & 180 & GRACE & $\begin{array}{l}\text { Based on GRACE inter- } \\
\text { satellite ranging and } \\
\text { SST } \\
\text { Mayer-Gürr et al. } \\
\text { (2010) }\end{array}$ \\
\hline EGM2008 & 2,190 & $\begin{array}{l}\text { GRACE, } \\
\text { G,A }\end{array}$ & $\begin{array}{l}\text { Based on ITG- } \\
\text { GRACE03S GGM, } \\
\text { altimetric and terrestrial } \\
\text { data } \\
\text { Pavlis et al. (2008) }\end{array}$ \\
\hline
\end{tabular}

GOCO01S (Pail et al. 2010a; GOCO Consortium 2010) is a combination of two months GOCE gradiometry data with seven years of GRACE observations (model ITGGRACE2010s, Mayer-Gürr et al. 2010). GOCO01S makes use of the complementary character of the two missions: GRACE delivers superior information on the low- and medium- degree gravity field constituents, coarsely up to degree 100 , while GOCE is particularly sensitive to the medium wavelengths, to degree 200 , with a comparable spectral performance found in spectral band from 130 to 150 (Pail et al. 2010a). The GOCO01S model is constrained by Kaula's (1966) rule of thumb (power-decay of gravity field signals with degree) in their upper bands.

The GOCE-DIR model (Bruinsma et al. 2010; Pail et al. 2011) uses the direct computation approach and incorporates the EIGEN-51C combined GGM (Bruinsma et al. 2010) as a background model for the regularisation of the GOCE model. EIGEN-51C is based on six years of CHAMP (Challenging Mini-satellite Payload; e.g., Reigber et al. 2002) 
and GRACE data and incorporates the DNSC08GRA global gravity anomaly data set (Andersen et al. 2010) that uses EGM2008 gravity over land. The regularisation has been done by applying the so-called spherical cap regularisation (Metzler and Pail 2005) with EIGEN-51C as pre-information over the polar caps. Due to this regularization approach, the GOCE-DIR model is 'reinforced' by EIGEN-51C. In the long wavelengths, EIGEN-51C utilises information from GRACE and CHAMP, while the medium- to short-wavelength information is sourced from DNSC08GRA (and hence EGM2008 terrestrial gravity over land areas). This is important to know for a correct interpretation of our evaluation results in Sect. 4. With the (restricted) information available to us, it is not possible to quantify to what extent GOCE observations and EIGEN-51C data contribute to the GOCE-DIR GGM in the various spectral bands.

GOCE-TIM (Pail et al. 2010b; Pail et al. 2011) is the only GGM that relies exclusively on GOCE observations (gradiometry and SST). Kaula (1966) regularisation is used to constrain the model's short wavelengths. Evaluation of GOCE-TIM (Sect. 4) provides pure feedback on the GOCE-only performance (as reached after two months) without influence of any other data sets.

The space-wise model GOCE-SPW (Migliaccio et al. 2010; Pail et al. 2011) not only uses the first two months of GOCE observations, but also EGM2008 (Pavlis et al. 2008) as apriori information to strengthen the model in the long wavelengths, which is effectively the same as using ITG-GRACE03S (Mayer-Gürr 2007). In the medium wavelengths, however, GOCE-SPW can be considered a GOCE-only model.

The two GRACE-based satellite-only GGMs included in our analysis are GGM03S (Tapley et al. 2007) and ITG-GRACE-2010S (Mayer-Gürr et al. 2010). These two are based on $\sim 4$ years and $\sim 7$ years of GRACE data, respectively, and are used as a 'benchmark' of preGOCE satellite gravimetry.

Finally, EGM2008 (Pavlis et al. 2008) serves as a source for omission error estimates for the SEM technique (Sect. 4). Importantly, EGM2008 is also used as a 'standard' over well-surveyed areas against which the performance of the new GOCE-solutions are tested (cf. Sects. 4 and 5). EGM2008 is a combined model based on ITG-GRACE03S (a predecessor of ITG-GRACE-2010S, cf. Mayer-Gürr 2007) and 5 arc minute surface gravity data from a variety of sources (Pavlis et al. 2008). EGM2008 delivers highly-accurate information of the spectral band 2 to 2190 and is widely considered as the current state-of-the-art of highresolution global gravity field modelling (e.g., Newton’s Bulletin 2009).

\subsection{Terrestrial comparison data}

Although the SEM can be applied fairly independently of the arrangement of the terrestrial (ground-truth) stations, some basic conditions should be met to facilitate a 'useful' GGM evaluation. First, it is preferable to use terrestrial data sets consisting of several 100 (or many more) stations, thus making the results of the RMS analysis reasonably independent of individual errors of the terrestrial observations and the SEM-modelled omission error corrections, as well as being statistically significant. Second, given the spatial resolution of the GOCE GGMs is $\sim 100 \mathrm{~km}$, it is desirable for the data sets to cover a larger area (country or even continental scale), to make the analysis spectrally representative. Finally, the comparison data should be independent of the GOCE-based GGMs. It is beneficial if the 
comparison data is also independent of the data sets sourced by the SEM technique to model the omission errors (here the EGM2008 GGM and RTM). All data sets described next are accompanied by accurate ellipsoidal heights (directly observed with GPS or constructed from spirit-levelling plus regional quasigeoid heights), as required for the computation of gravity disturbances from observed gravity and harmonic synthesis (cf. Sect. 2.3).

\subsubsection{Terrestrial gravity}

The present study utilises the national gravity data set of Switzerland (Marti 2004) and a selected subset of gravity stations from the Australian national gravity data base (Murray 1997). The Swiss data set consists of 31,598 stations (release of 2003) and was kindly provided by swisstopo (national surveying agency of Switzerland). The gravity station coverage (Fig. 2) extends over the whole of Switzerland including all types of topography (from the low-elevated Northern parts of Switzerland to the rugged Swiss Alps in the South). Subsets also cover adjacent regions of neighbouring countries. The accuracy of the Swiss gravity is at the $0.1 \mathrm{mgal}$ level or better (U. Marti, pers. comm. 2010). With an area of roughly $200 \mathrm{~km}$ x $350 \mathrm{~km}$ covered, this data set provides a sufficiently large area to provide feedback on the GOCE models.

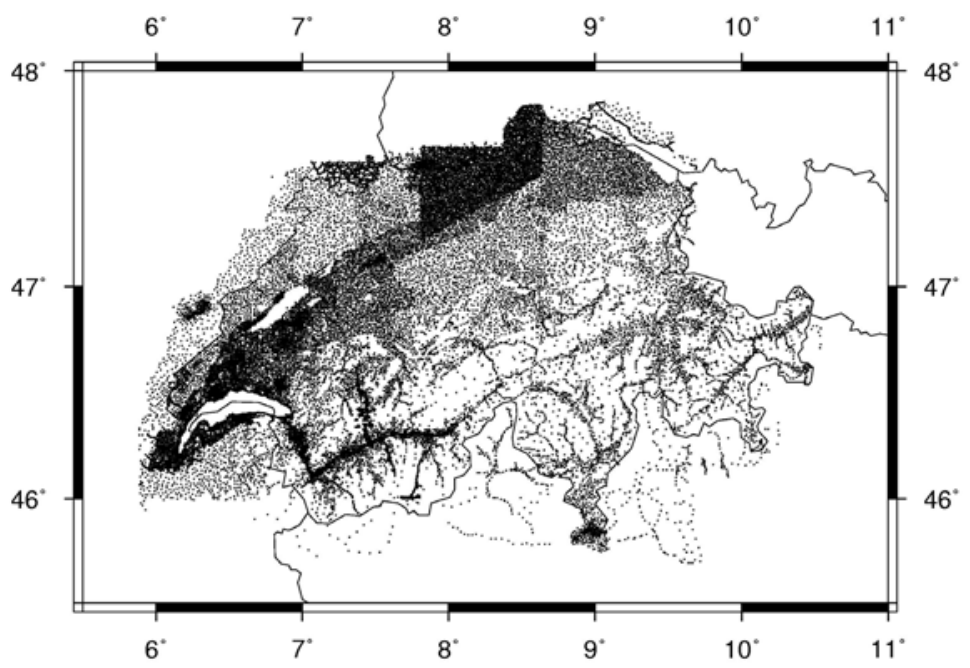

Fig. 2 Location of 31,598 gravity stations over Switzerland 


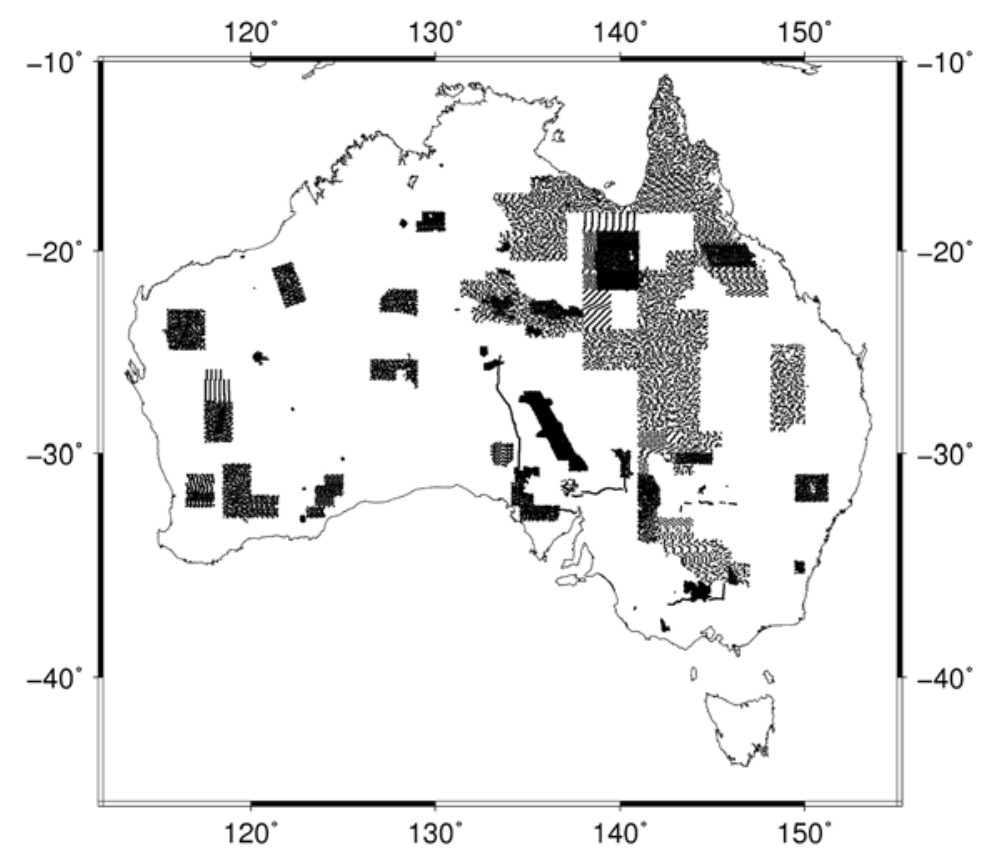

Fig. 3 Location of 28,551 recent gravity stations over Australia

As second set, we selected a subset of 28,551 gravity stations over Australia (Fig. 3), acquired during the last decade, from the Geoscience Australia (GA) land gravity data base (http://www.gadds.ga.gov.au), which consists of roughly 1.5 million observations (end of 2010). Most of the 28,551 stations were collected in areas of resource prospectivity in dense grids (visible as 'patches' in Fig. 3) with a spatial resolution of 2-4 km (Featherstone et al. 2011). As opposed to many poorly documented 'historical' gravity sets held in the GA land gravity data base, the selected data is coordinated with GPS and at the $0.1 \mathrm{mGal}$ accuracy level, so it can be considered to be sufficiently reliable. There exist another $\sim 250,000$ GPScoordinated gravity stations in the GA data base, but these were not included because they do not cover other areas than those shown in Fig. 3. Though mostly arranged in patches (Fig 3), the selected data extends over an area of coarsely $\sim 2000 \mathrm{~km} \mathrm{x} \sim 3000 \mathrm{~km}$. Covering a large part of Australia as a significant land mass in the Southern Hemisphere, we consider this data set to play an important role in the evaluation of the new GOCE data sets. The inspection and inclusion of further extracts of the GA land gravity data base is intended as a future task.

\subsubsection{Vertical deflections}

Sets of astrogeodetic vertical deflections are available over Europe and Australia for this study. The European vertical deflection set (Fig. 4) originates exclusively from zenith camera observations (e.g., Bürki et al. 2007, Hirt et al. 2010c). It consists of 1,056 astrogeodetic stations observed with analogue zenith cameras (433 stations) and digital zenith cameras (623 stations) over the last three decades. The most important part of the European vertical deflections are 540 stations covering the whole of Switzerland (provided by swisstopo and the Swiss Geodetic Commission). Other subsets of digital zenith camera measurements cover parts of Portugal and Greece (provided by B. Bürki, ETH Zürich), the Bavarian Alps (Hirt and Flury 2008), parts of Northern Germany and the Netherlands. We refer to Hirt et al. (2010a) for full details on this data set. The accuracy of the digital zenith camera vertical 
deflections is 0.1" (Hirt and Seeber 2008) while the data sets originating from analogue observations are accurate to 0.3"-0.5" (Hirt et al. 2010a, Hirt et al. 2010c).

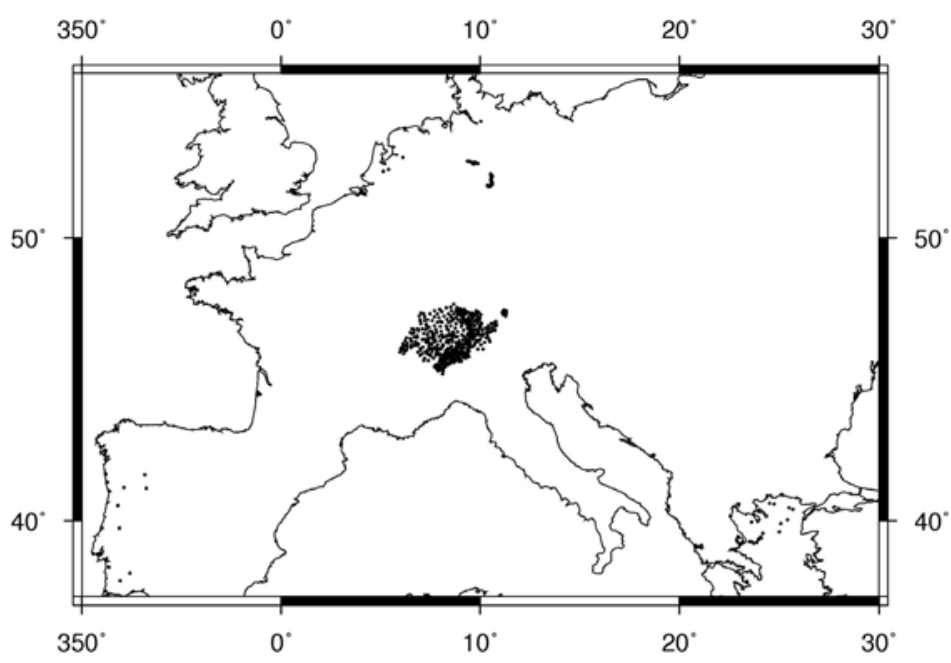

Fig. 4 Location of 1,056 vertical deflections over Europe

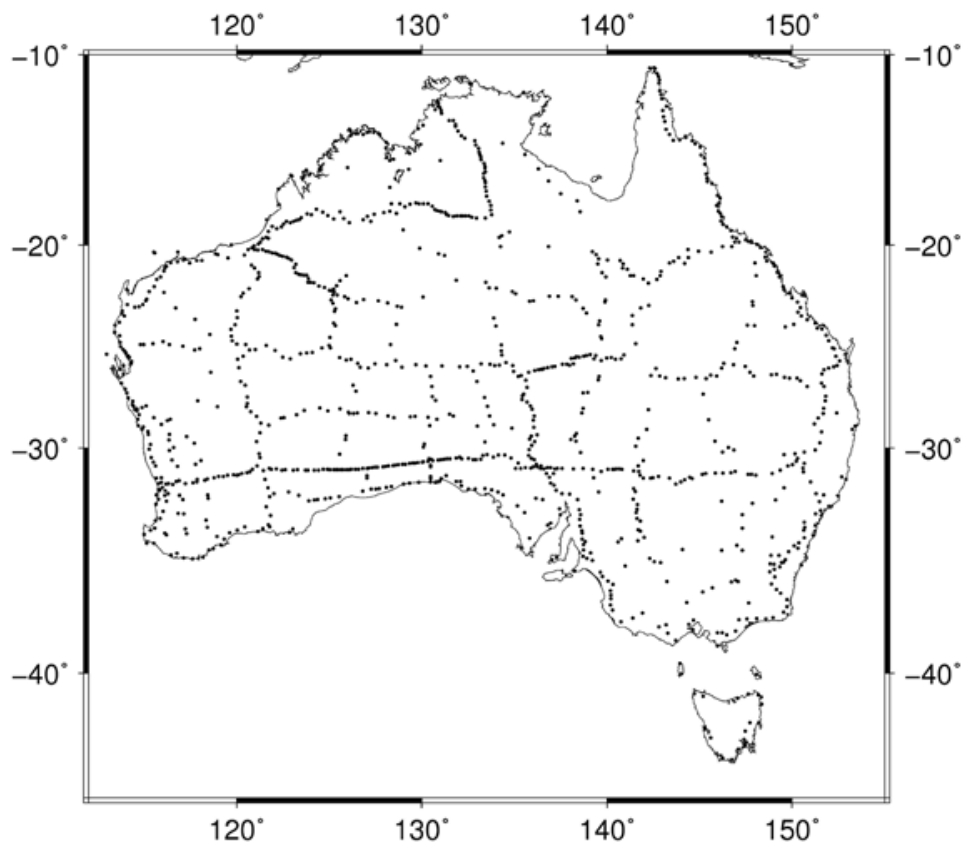

Fig. 5 Location of 1,064 vertical deflections over Australia

The 1,056 European vertical deflections is the largest astrogeodetic data set available from zenith camera observations so far. Though the station coverage is not optimum for the purpose of GGM validation (e.g., parts of the European continent remain uncovered, subsets of vertical deflections are arranged locally along densely-spaced profiles), the set was already used for an assessment of EGM2008 (Hirt et al. 2010a). This study demonstrated the suitability of the European deflection data set for GGM evaluation. Another set of $\sim 300$ vertical deflections (not used here) was recently observed for GOCE validation with a digital zenith camera in Germany. These stations, located along two $500 \mathrm{~km}$ profiles, were low- 
pass filtered with a Gaussian filter for comparisons with recent GGMs (Voigt et al. 2009; Ihde et al. 2010).

Secondly, a set of 1,080 astrogeodetic vertical deflections is used over Australia, provided by GA and Landgate (the Western Australian State geodetic agency). This set covers the whole of the Australian continent (Fig. 5). The Australian vertical deflection data set originates from 'historic' observations, carried out around four decades ago (Featherstone and Morgan 2007; Featherstone and Lichti 2009). The accuracy of the Australian vertical deflections has been "cautiously estimated to be 1 arc second" (Featherstone and Lichti 2009). Comparison of the Australian vertical deflections with EGM2008/RTM-based vertical deflections revealed a total of 16 clear outliers (discrepancies larger than 6 arc seconds), which were removed prior to its use for GOCE model evaluation. Though not as accurate as the European set, our experiments will demonstrate that the Australian deflections are capable of providing some valuable feedback on the new GOCE models (Sect 4.2).

\section{Evaluation results}

From our terrestrial comparison data, only the vertical deflections are independent of all GGMs used here and therefore suited to identify - in comparison to a pure EGM2008/RTM solution (Fig. 1C) - if and in which spectral bands the first GOCE models improve the gravity field in our test areas. As opposed to this, large parts of the Swiss and Australian gravity data were used in the construction of EGM2008. As an immediate consequence, the EGM2008/RTM solution is 'biased' towards the gravity data and not suited to serve as a 'fair benchmark' for evaluation of the new GOCE-based data sets in our SEM scheme. Also, the GOCE-DIR model is not independent from the terrestrial gravity data sets because of the use of EIGEN-51C in the model development (cf. Sect. 3). In case of existing inter-dependencies, however, the relative comparison between the RMS curves is used to determine the actual spectral content of the new GOCE models.

The subsequent analyses are based on the SEM variants shown in Figs. 1A and 1C. The statistics of the differences between the 'observed' gravity disturbances (Sect. 2.3, step 1) and the model values from EGM2008 (band 2 to 2190) and EGM2008 (band 2 to 2190) augmented by RTM (Fig. 1C) are reported in Table 2. The RTM augmentation reduces the RMS errors to $\sim 4.5 \mathrm{mGal}$ for the Swiss data ( $\sim 89 \%$ reduction) and to $\sim 2.7 \mathrm{mGal}$ for the Australian data ( $\sim 21 \%$ reduction). For the sake of completeness, we also list the descriptive statistics of the differences 'observed deflection minus model deflection' (Table 3). By virtue of RTM augmentation, the RMS errors are reduced to the 1 arc second level and below ( $65 \%$ reduction over Europe, $\sim 10 \%$ over Australia). The variations of the RMS differences due to using GOCE-based GGMs instead of EGM2008 in the spectral window $2 \ldots \mathrm{n}_{1}$ are analysed in Sects. 4.1 and 4.2 .

The relatively low RMS values of the differences 'observation minus EGM2008/RTM' (Tables 2 and 3) indicates a reasonably good quality of the terrestrial data and the SEM-based omission error modelling. It should be noted that the RMS values reflect any errors of the data sets involved, so the EGM2008 commission errors in the range $n_{1}$ to 2190, commission errors in the RTM corrections (e.g., due to unmodelled mass-density anomalies) and observational errors of the terrestrial data sets. Because of the presence of 
these error sources, the use of the new GOCE-GGMs (in place of EGM2008) is not expected to lead to a significant decrease of the RMS values listed in Tables 2 and 3.

Table 2 Descriptive statistics of the gravity disturbance differences observation minus model, units in mGal.

\begin{tabular}{|l|l|l|r|r|r|r|}
\hline Area & \#points & Differences & \multicolumn{1}{l|}{ Min } & \multicolumn{1}{l|}{ Max } & \multicolumn{1}{l|}{ Mean } & \multicolumn{1}{l|}{ RMS } \\
\hline \multirow{3}{*}{ Switzerland } & 31,598 & Obs - EGM2008 & -224.70 & 95.09 & -18.18 & \pm 39.50 \\
\cline { 2 - 7 } & 31,598 & Obs - EGM2008/RTM & -98.92 & 32.31 & -1.57 & \pm 4.48 \\
\hline \multirow{2}{*}{ Australia } & 28,551 & Obs - EGM2008 & -34.70 & 34.43 & -1.04 & \pm 3.40 \\
\cline { 2 - 7 } & 28,551 & Obs - EGM2008/RTM & 22.12 & 24.93 & -0.92 & \pm 2.68 \\
\hline
\end{tabular}

Table 3 Descriptive statistics of the vertical deflection differences observation - model, units in arc seconds. $\mathrm{xi}=$ North-South vertical deflection component, eta $=$ East-West vertical deflection component. 16 outliers were removed from the Australian vertical deflection set.

\begin{tabular}{|l|l|l|c|r|r|r|c|}
\hline Area & \#points & Differences & Comp. & \multicolumn{1}{l|}{ Min } & \multicolumn{1}{c|}{ Max } & \multicolumn{1}{l|}{ Mean } & \multicolumn{1}{l|}{ RMS } \\
\hline \multirow{5}{*}{ Europe } & 1,056 & Obs - EGM2008 & xi & -15.00 & 15.54 & -0.03 & \pm 3.02 \\
\cline { 2 - 8 } & 1,056 & Obs - EGM2008/RTM & xi & -4.74 & 5.37 & -0.04 & \pm 1.04 \\
\cline { 2 - 8 } & 1,056 & Obs - EGM2008 & eta & -11.67 & 15.62 & 0.32 & \pm 2.97 \\
\cline { 2 - 8 } & 1,056 & Obs - EGM2008/RTM & eta & -4.33 & 4.90 & 0.15 & \pm 1.05 \\
\hline Australia & 1,064 & Obs - EGM2008 & xi & -4.89 & 3.33 & -0.15 & \pm 0.78 \\
\cline { 2 - 8 } & 1,064 & Obs - EGM2008/RTM & xi & -4.93 & 2.62 & -0.15 & \pm 0.67 \\
\cline { 2 - 8 } & 1,064 & Obs - EGM2008 & eta & -4.98 & 3.78 & -0.09 & \pm 1.03 \\
\cline { 2 - 8 } & 1,064 & Obs - EGM2008/RTM & eta & -5.24 & 4.14 & -0.10 & \pm 0.96 \\
\hline
\end{tabular}

To identify the spherical harmonic degree where the new GOCE-GGMs lose spectral power (denoted here as signal loss), we introduce an arbitrary threshold

$$
\text { th }_{\text {signal_loss }}=1.1 \cdot \operatorname{RMS}(\text { EGM2008only), }
$$

i.e., a threshold value that is $10 \%$ larger than the RMS value of the differences 'observation EGM2008-only solution'. Visually, signal loss manifests as steep slopes of the RMS curves shown in Figs. 6 to 9. The RMS values are computed as a function of $n_{1}$ (ranging from 2 to

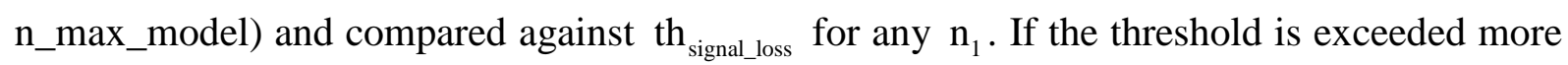
than once, we consider the smallest value $n_{1}$ to be the spherical harmonic degree of signal loss under our criterion. Next, the RMS curves are presented and discussed for our four test areas. The spherical harmonic degrees of signal loss, as obtained from the numerical criterion in Eq. (1) are reported in Table 4.

\subsection{Results using terrestrial gravity}

Figure 6 visualises the SEM evaluation results using the Swiss gravity data. The RMS values of the gravity disturbance differences 'observation minus model' are shown as a function of the spherical harmonic band 2 to $\mathrm{n}_{1}$ and the seven GGMs evaluated (Table 1). All the GGMs 
show comparable performance up to degree 140. GGM03S (green-solid line) and ITGGRACE2010S (green-dotted line) experience signal loss at degree 142 and 170, respectively. The signal loss is seen by the rapidly increasing RMS values in Fig 6.

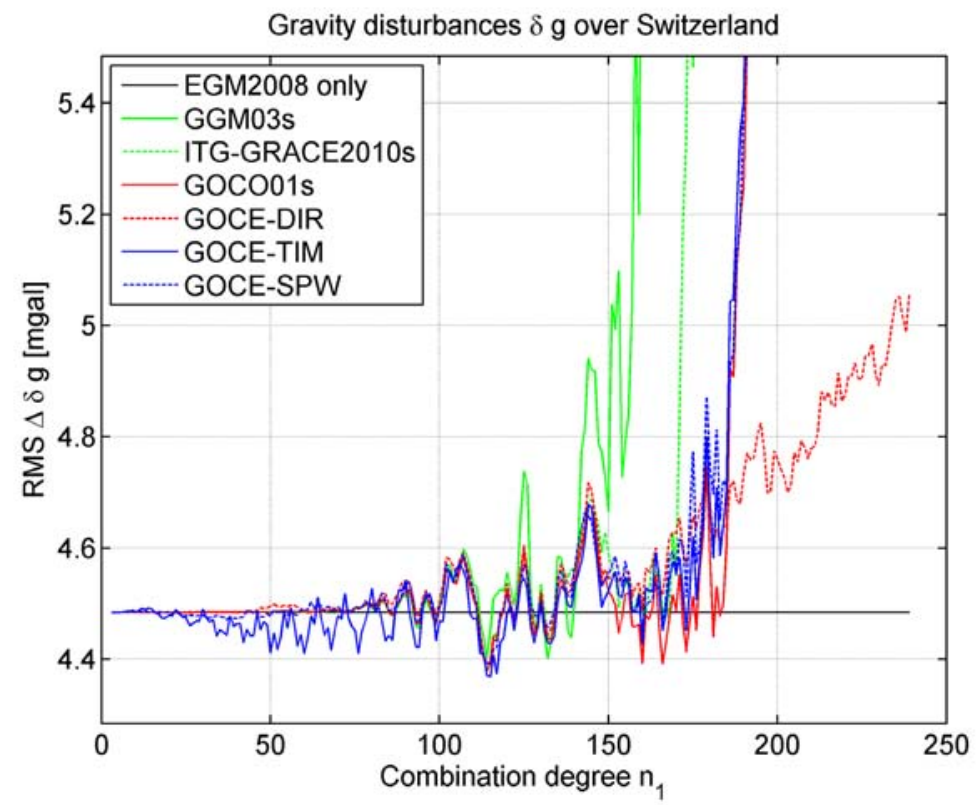

Fig. 6 RMS of the gravity disturbance differences over Switzerland as a function of the GGM used in spectral window $2 \ldots \mathrm{n}_{1}$, Units in $\mathrm{mGal}$.

In contrast, steep slopes are visible in Fig. 6 for the GOCE models GOCO01S (redsolid line), GOCE-TIM (blue-solid line) and GOCE-SPW (blue-dotted line) near degree 185 (Table 4). This shows that the GOCE-based models improve knowledge in the spectral band $\sim 170$ to 185 with respect to GRACE. For GOCE-DIR (red-dotted line), a similarly sudden signal loss cannot be observed over the whole spectral band. However, this is not necessarily attributable to GOCE, because EIGEN-51C terrestrial gravity anomalies were used to reinforce the GOCE-DIR model in the high-degrees. The somewhat worse performance of GOCE-DIR in comparison to EGM2008 in the short wavelengths (to degree 240) could be related to the increasing noise of GOCE observations, but this cannot be proven with the data available to us. It should be noted that oscillations of the RMS values are visible all over the low and medium and short degrees with varying amplitudes (cf. Sect. 4.3). 


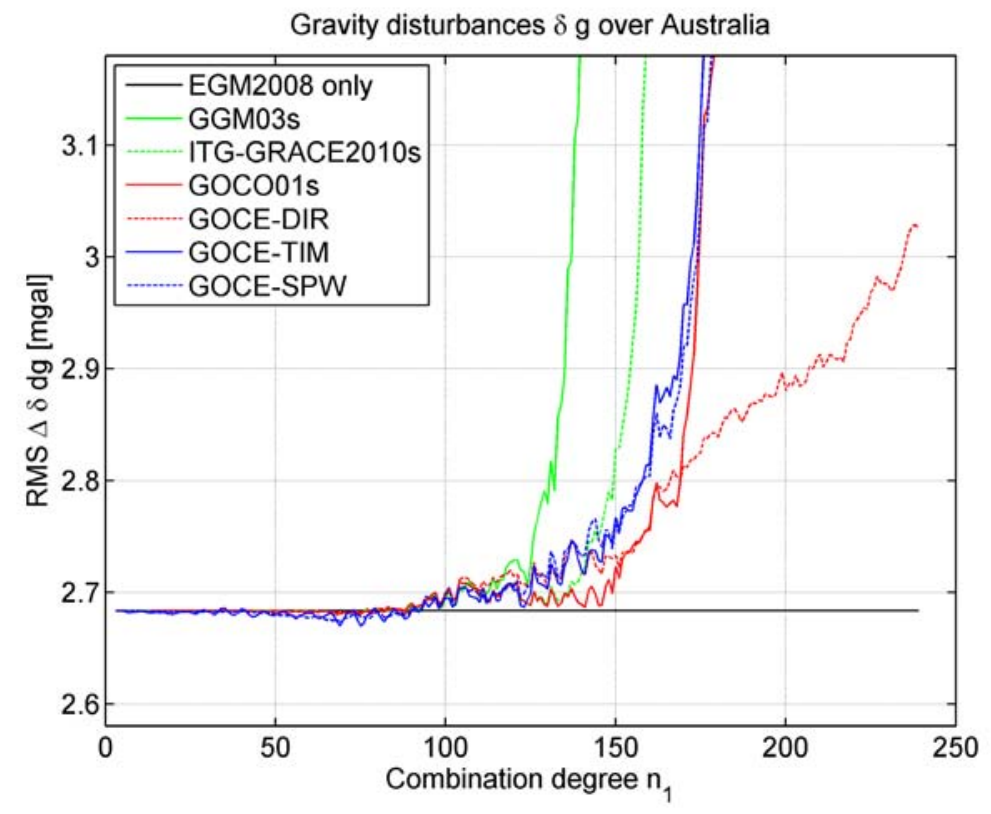

Fig. 7 RMS of the gravity disturbance differences over Australia as a function of the GGM used in spectral window $2 \ldots \mathrm{n}_{1}$, Units in $\mathrm{mGal}$.

The SEM evaluation results are shown in Fig. 7 for the Australian gravity data set. In comparison to the Swiss data, the models experience signal loss roughly 15 spherical harmonic degrees 'earlier', e.g. ITG-GRACE2010S near degree 154 instead of 170 and the GOCE-based GGMs GOCO01S, GOCE-TIM and GOCE-SPW at degree 170 instead of 185 (see Table 4). Despite this somewhat enigmatic shift, which may be due to the uncertain GPS reference frames used to coordinate the gravity surveys (Featherstone et al. 2011), it can be seen that the three GOCE-based models provide improved information on the band $\sim 155$ to $\sim 170$ in comparison to the GRACE-only GGMs. Again, GOCE-DIR experiences only a slowly progressing signal loss in its upper band $\sim 170$ to 240 , which is explained by the terrestrial data included in its construction.

Both over Switzerland and Australia, EGM2008 exhibits for most of the spectral degrees the 'best' RMS performance. However, this should not be over-interpreted, recalling the inter-dependencies between EGM2008 and the terrestrial gravity data. The observed similar behaviour of those GGMs using GOCE-only data in their upper bands (GOCO01S, GOCE-TIM and GOCE-SPW) suggest a very comparable performance of the underlying GOCE computation methods ‘time-wise-approach' and 'space-wise-approach'. For all of the GGMs involved, a fairly similar performance is observed for the long wavelengths (degrees 2 to $\sim 100$ ).

\subsection{Results using vertical deflections}

Figure 8 shows the RMS values of the vertical deflection differences 'model minus observation' for our European data set as a function of the GGM used in spherical harmonic band 2 to $n_{1}$. Again, the SEM technique (Sect. 2 and Fig. 1A) was applied to reduce the discrepancies between the data sets as much as possible. From Fig 8A (North-South vertical deflection component xi), the GRACE-only models GGM03S and ITG-GRACE2010S experience signal loss between degrees $\sim 160$ to $\sim 170$. GOCO01S, GOCE-TIM and GOCE- 
SPW exhibit signal loss near degree 200 , which suggests superior GOCE performance in band $\sim 170$ to almost $\sim 190$ to $\sim 200$. The GOCE-DIR model does not experience any signal loss, as is seen in Fig. 8A. The RMS values of the GOCE models in spectral window $\sim 150$ to $\sim 190$ are mostly smaller than those of EGM2008 (red line). Recalling that our vertical deflections are independent of all GGMs used, this indicates an improved gravity field knowledge due to the new GOCE models even in a densely and well-surveyed area such as Europe.

Analysis of the vertical deflection residuals in the East-West direction (eta component) does not give a similarly clear picture (Fig. 8). The steep gradients for the GRACE-based models are further apart (degrees 145 for GGM03S and $170-175$ for the ITG model), with the latter not clearly separated from slopes of the GOCE models (around 185). Thus, the eta-component shows only a slight improvement of gravity field knowledge in the according bands. In the spectral band 100-150, the RMS values of the GOCE-based models are somewhat smaller than those of the GRACE-only models and EGM2008.
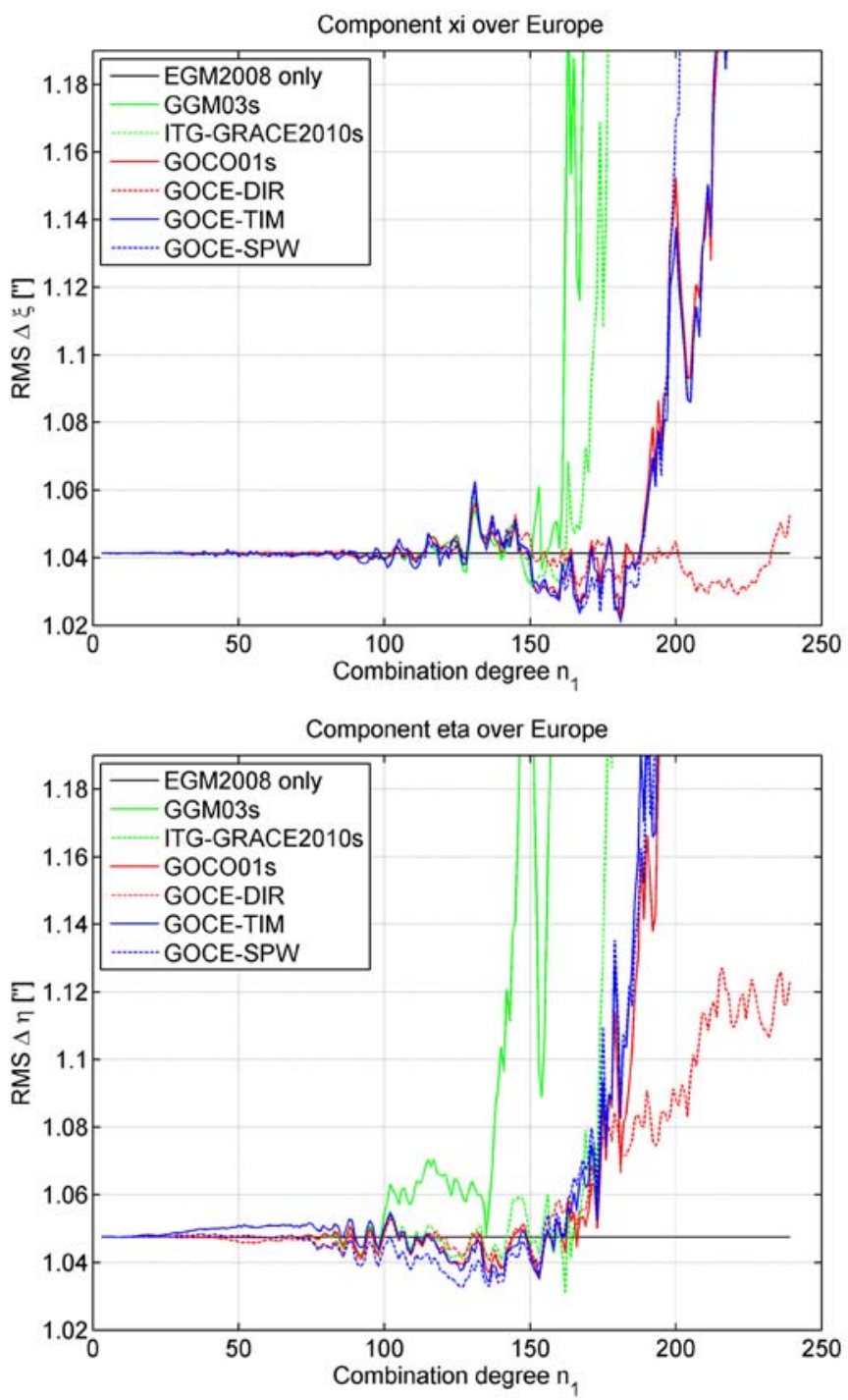

Fig. 8 RMS of the vertical deflection differences over Europe as a function of the GGM used in spectral window $2 \ldots n_{1}$. Top: Vertical deflection component xi, Bottom: component eta. Units in arc seconds. 

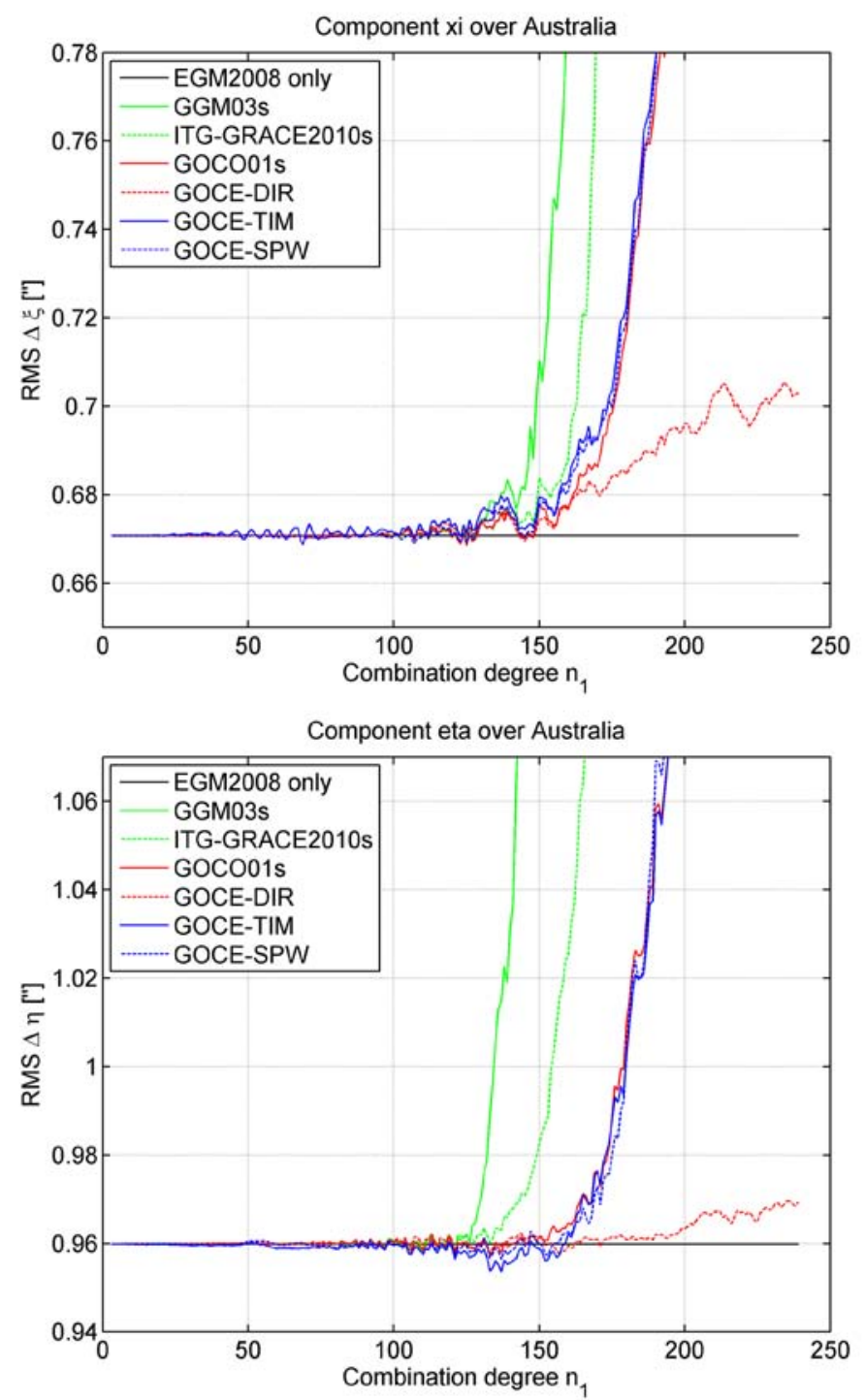

Fig. 9 RMS of the vertical deflection differences over Australia as a function of the GGM used in spectral window $2 \ldots \mathrm{n}_{1}$. Top: Vertical deflection component xi, Bottom: component eta. Units in arc seconds.

Focussing on the [less accurate] Australian vertical deflections (Fig. 9), the GOCEbased models GOCO01S, GOCE-TIM and GOCE-SPW experience signal loss near spherical harmonic degree $\sim 180$-190 (both for xi and eta). Opposed to this, the GRACE-only model ITG-GRACE2010S shows steep slopes near degree 165. The EIGEN-51C augmented GOCE-DIR model shows a similar behaviour and, additionally, only a slight deterioration in their RMS values in the spectral band 180 to 240. This again demonstrates improvements conferred by GOCE in the spectral window $\sim 165$ to $\sim 185$. It is remarkable that the $\sim 40+$ year old Australian vertical deflections 'see' the advancements in gravity field knowledge coming from only two months of GOCE observations. As such, old and seemingly inaccurate 'ground-truth' data (but if in sufficient quantity) can still be useful for GGM validation. 


\subsection{Synthesis of results}

From the range of observations made in the GGM comparisons (Table 4), we can surmise the following: our gravity observations and vertical deflection data sets are capable of sensing improvements delivered by the new GOCE gravity field models, even if they are based only on two months of in-orbit data. Over our ground-truth areas, the first four GOCE models advance knowledge of the Earth's static gravity field. Depending on the gravity field functional and test area, the degrees of the improved spectral band vary, but it can be broadly stated that the improved spectral band begins near degree $160-165$ and ends near degree 180-190 (Table 4).

Table 4 Actual model resolution (as indicated by the ground-truth comparisons). The listed values are the spherical harmonic degrees where the models experience signal loss as computed with the numerical criterion in Eq. (1) n.o. = not observed.

\begin{tabular}{|l|l|l|l|l|}
\hline GGM $\backslash$ Dataset & $\begin{array}{l}\text { Swiss } \\
\text { Gravity }\end{array}$ & $\begin{array}{l}\text { Australian } \\
\text { Gravity }\end{array}$ & $\begin{array}{l}\text { European } \\
\text { deflections } \\
\text { xi, eta }\end{array}$ & $\begin{array}{l}\text { Australian } \\
\text { deflections } \\
\text { xi, eta }\end{array}$ \\
\hline GOCO01s & 186 & 172 & 198,186 & 182,188 \\
\hline GOCE-DIR & 224 & 221 & n.o. & n.o. \\
\hline GOCE-TIM & 184 & 168 & 209,185 & 180,188 \\
\hline GOCE-SPW & 185 & 170 & 197,186 & 181,188 \\
\hline GGM03s & 142 & 134 & 161,144 & 153,140 \\
\hline ITG-GRACE2010s & 170 & 154 & 172,174 & 166,162 \\
\hline
\end{tabular}

The variations in the model resolutions (Table 4) suggest the possibility of a hemisphere-dependent performance of GOCE (cf. European and Australian results). However, additional results from the second assessment technique using global EGM2008 quasigeoid grids do not indicate a worsened GOCE performance over the Southern Hemisphere continents (Sect. 5). Therefore, the observed variations in the resolution of the GOCE models (Table 4) are attributed to the test data sets (e.g., station distribution, area covered) and not to GOCE. This finding is also considered a justification to use different ground truth areas and functionals holistically, so as to obtain a better picture of the actual model signal content.

From any of our comparisons, comparable performance is observed for GOCO01S, GOCE-TIM and GOCE-SPW over the whole spectrum. Significant differences among these methods are not indicated by our comparisons, and they are unable to discriminate between them. Finally, the GOCE-DIR model outperforms the other three-GOCE-based models in the spectrum beyond $\sim 190$, where it loses only little spectral power. This is expected as it is augmented with terrestrial data, whereas Kaula's (1966) rule of thumb dictates that satelliteonly GGMs cannot sense the high-frequency gravity field. The GOCE-DIR performance must be balanced against the fact that the EIGEN-51C model is used as reinforcement and, hence, the main data source in this short-wavelength window is assumed to be terrestrial gravity. As a consequence, there is no 'fair' comparison possible among the performance of 
the direct computation approach and the time-wise or space-wise approach to GOCE gravity field recovery.

The terrestrial gravity data sets (Figs. 6 and 7) showed a slightly better or equal performance of GOCO01S with respect to GOCE-TIM and GOCE-SPW for spectral degrees $\sim 100$ and beyond. With the exception of vertical deflection component eta over Australia, this is also seen from the vertical deflection comparisons (Fig. 8 and 9). These observations indicate that the combined GOCE/GRACE gravity field modelling, as described by Pail et al. (2010a) yields more accurate models of Earth's gravity field than GOCE alone.

Our comparisons involving vertical deflections show for any of the new GOCE-based GGMs (and for ITG-GRACE2010S) RMS values which are mostly similar and often smaller than those of the EGM2008-only/RTM solutions (Fig. 8 to 10). Given that vertical deflections are truly independent, this indicates a tendency of the new GOCE models (and the ITG model) having more than comparable quality than EGM2008 in the tested spectral bands.

Finally, all RMS curves in Figs. 6 to 9 are subject to oscillations with a wavelength of coarsely $\sim 10$ spherical harmonic degrees. Striking examples are Fig. 6 (Swiss gravity), spherical harmonic degree 145, Fig 8A (European deflections, component xi), degrees 135 and 175 and many more occurrences are visible over the whole range of $n_{1}$-combination degrees. Given that the RTM contributions are always the same, these oscillations provide some evidence that the spectral enhancement of GGMs (use of the GGM to degree $n_{1}$ ) with EGM2008 (use of EGM2008 from $\mathrm{n}_{1}+1$ onwards) is not a rigorous technique (see also Featherstone 2007 p. 76). A discussion of the mechanisms that cause these oscillations is beyond the scope of the present study.

\section{Comparisons using global EGM2008 quasigeoid heights}

A characteristic of the 'ground-truth' comparisons presented in Sect. 4 is that the comparison data sets are restricted to the geographical regions of some limited extent. Although the above experiments justify the assumption that the GOCE models improved knowledge in the spectral band 165-185 globally (and not only over our test areas), an alternative EGM2008based assessment technique is used, serving to support the SEM-based ground-truth comparisons in Sect. 4.

\subsection{Concept}

The basic idea of the EGM2008-based assessment is to compare global grids of quasigeoid heights (other functionals or the disturbing potential itself could be used) from EGM2008 and GOCE in identical spectral bands, e.g., 2 to 180. The omitted signals (beyond the band's upper degree) should be similar, so largely cancel in the quasigeoid differences between EGM2008 and GOCE. Therefore, no omission error modelling is required when comparing identical bands.

Importantly, the comparisons between EGM2008 and GOCE GGMs under evaluation are facilitated by the global distribution of the EGM2008 commission errors (EGM Development Team 2009) derived from an error propagation technique described in Pavlis and Saleh (2004). Figure 10 shows the EGM2008 quasigeoid commission error as a function of the geographic location (data from EGM Development Team 2009). Though Pavlis et al. (2008) computed the commission errors for spectral band 2 to 2160, we assume that Fig. 10 
provides a good enough indication on the global distribution of EGM2008 commission errors for low/medium degree spectral bands (e.g., 2 to 180). This is justified by the fact that most of the spectral energy of quasi/geoids are in the lower and medium degrees (Torge 2001, p. 274f; Schwarz 1985).

Figure 10 shows that EGM2008 quasigeoid heights are predicted to be accurately known $(\sim 5 \mathrm{~cm})$ over most parts of Europe, North America and Australia, where dense and available terrestrial gravity data sets were used in EGM2008 (Pavlis et al. 2008). Over these three continents, and the oceans, we consider EGM2008-quasigeoid heights to have 'groundtruth' quality for the purpose of GOCE model evaluation. In contrast to this, commission errors of $\sim 15 \mathrm{~cm}$ and larger (Fig. 10) reveal large parts of Asia, Africa, South America and Antarctica as regions with poor, restricted or no terrestrial gravity data coverage, and where the EGM Development Team had to use 'fill-in' anomalies (Pavlis et al. 2007; Pavlis et al. 2008). As such, over large parts these continents, EGM2008 quasigeoid heights do not represent decent ground truth.

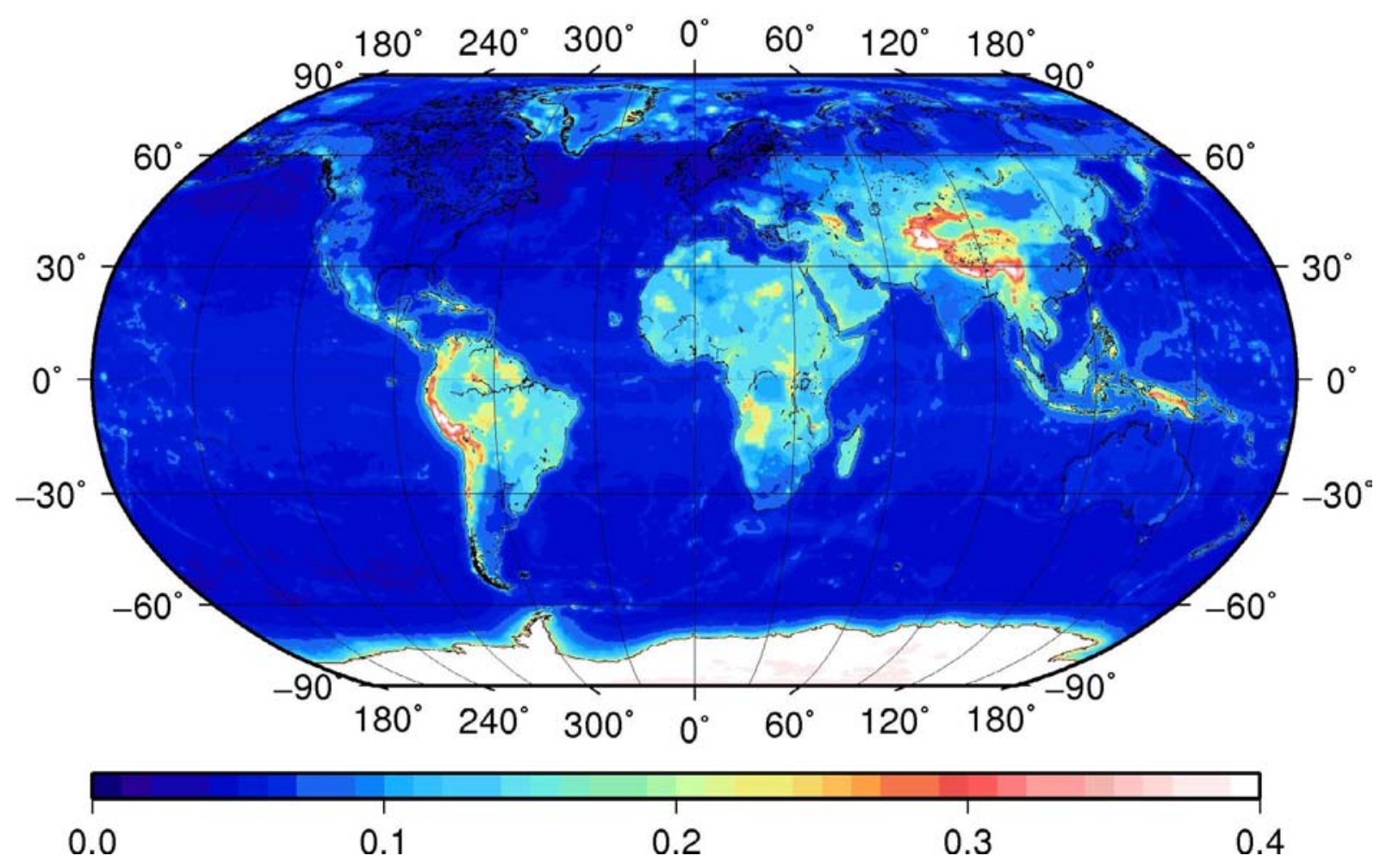

Fig. 10 EGM2008 quasigeoid height commission error, unit is metres 
The global map of EGM2008 commission errors (Fig. 10) represents valuable background information for GOCE model evaluation from EGM2008-GOCE residuals. This is because the commission error knowledge allows discrimination between EGM2008 and GOCE errors by establishing following simple three-case-scheme for interpretation of EGM2008 minus GOCE differences:

- Small differences, occurring over regions with low EGM2008 commission errors (e.g., Europe), indicate that two months of GOCE is already competitive with EGM2008.

- Large differences, occurring over regions with significant EGM2008 commission errors (e.g., Himalaya) indicate GOCE may improve over EGM2008. However, since there are no ground truth data in these regions, it is only possible to make an inference.

- High-frequency difference patterns (cantaloupe effect) occurring homogenously over the whole of the Earth and becoming more pronounced with larger truncation degree do not relate to EGM2008 quality. Instead, they are attributable to GOCE and reflect the degree of signal loss (cf. Fig. 6 to 9).

\subsection{Results}

We computed global grids of quasigeoid heights (as point values) at a resolution of 20 arc min (540 x 1080 grid points) from all GGMs listed in Table 1 and for spectral bands 2 to 5, 2 to 10 up to 2 to n_max_model (e.g., band 2 to 210 in case of GOCE-SPW). Using identical spectral bands, quasigeoid differences between EGM2008 and each GOCE model and EGM2008 and the two GRACE-based models were computed. Six selected results are presented and interpreted next.

Figure 11 (top) shows the quasigeoid differences between EGM2008 and the pure GOCE model GOCE-TIM in bands 2 to 180. The descriptive statistics of the differences are reported in Table 5 for various regions, e.g., Earth, land and oceans, continents and selected areas of Asia, Africa and South America, and, additionally, without the polar areas not measured by GOCE (i.e., $|\varphi| \geq 83.3^{\circ}$ ) due to GOCE's inclination of $96.7^{\circ}$.

- A good mutual agreement between both models is observed over most areas where EGM2008 quasigeoid heights possess 'ground-truth quality', i.e., Europe (RMS of 7 $\mathrm{cm}$ ), Australia $(8 \mathrm{~cm})$, North America $(8 \mathrm{~cm})$ and most parts of the oceans (RMS of 10 $\mathrm{cm}$, Table 5).

- Large discrepancies are found over the EGM2008 'problem regions', these are Antarctica (RMS of $23 \mathrm{~cm}$ ), South America (RMS of $34 \mathrm{~cm}$, and $40 \mathrm{~cm}$ over the Andes/Amazonas Basin region), Asia (19 cm, going up to RMS of $42 \mathrm{~cm}$ and maximum difference of $\sim 2$ to $3 \mathrm{~m}$ over the Himalaya region) and Africa $(23 \mathrm{~cm}$, going up to $30 \mathrm{~cm}$ over Central Africa), cf. Table 5 and Fig. 11 (top).

Both observations almost perfectly correlate with the EGM2008 commission error map in Fig. 10. Together, this provides strong evidence of GOCE adding to knowledge of the Earth's gravity field over wide parts of Antarctica, South America, Africa and Asia, while simultaneously confirming the gravity field knowledge over the EGM2008 'high-quality' areas. A rigorous proof of improvements over the EGM2008 'problem' areas, however, would require ground-truth data, which are not available to us.

Further to this, the difference patterns in Fig. 11 show a homogeneous GOCEperformance over both hemispheres, which is an important observation in view of the 
expected improvements of gravity field knowledge for all Southern-Hemisphere continents (see also Sect 4.3). Figure 11 (bottom) shows the EGM2008 and GOCE-TIM quasigeoid residuals in spectral band 2 to 210. In comparison to Fig. 11 (top), additional high-frequency patterns appear over all continents and oceans, increasing the RMS differences, e.g., from 7 to $15 \mathrm{~cm}$ (Europe), 8 to $15 \mathrm{~cm}$ (Australia) and 10 to $17 \mathrm{~cm}$ (oceans); see Table 5. This shows the attenuation of GOCE-captured gravity field signals in bands 181 to 210. As an aside, Fig. 11 exhibits large differences for the polar regions (which cannot be observed due to GOCE's orbit inclination) and discrepancies between GOCE-TIM and EGM2008 over the Southern Ocean $\left(\sim 140^{\circ} \mathrm{E}, \sim 50^{\circ} \mathrm{S}\right)$, near the South Magnetic Pole, which have been found to be related to the GOCE yy-gravity-gradients (Pail 2011, pers. comm.).

Figure 12 (top) shows the EGM2008 and seven-year GRACE-only ITGGRACE2010S quasigeoid differences in bands 2 to 160 (see Table 6 for the descriptive statistics). The difference patterns bear strong resemblance with those from EGM2008 minus GOCE-TIM (Fig. 11 top), revealing the ITG-GRACE2010S improvements delivered for the EGM2008 'problem regions'. Figure 12 (bottom) shows the EGM2008 minus ITGGRACE2010S differences in bands 2 to 180. From Table 6, the RMS increases from $8 \mathrm{~cm}$ to $24 \mathrm{~cm}$ over the oceans and from $7 \mathrm{~cm}$ to $20 \mathrm{~cm}$ over Europe and North America. The noisepatterns visible in Fig. 12 (bottom) reflect GRACE signal loss in bands 161 to 180; also see Table 6.

The cross-comparison between Fig. 11 (GOCE-only results) and Fig. 12 (GRACEonly results) demonstrates improvements of GOCE with respect to the latest GRACE gravity field in bands $160 \ldots 180$ over the well-surveyed regions (seen by the good agreement with EGM2008). Additionally, the cross-comparison provides strong evidence that the two months of GOCE observations have delivered new information in bands 160...180 (and slightly beyond) over the poorly-surveyed regions of our planet.

Finally, the comparison between EGM2008 and GOCO01S (Fig. 13 and Table 7) shows in bands $2 \ldots 180$ and $2 \ldots 210$ slightly smaller residuals than the comparison between EGM2008 and GOCE-TIM (Fig 11). For bands 2...180, the RMS values drop, e.g., for Australia and North America from $8 \mathrm{~cm}$ to $7 \mathrm{~cm}$, and for Greenland from $12 \mathrm{~cm}$ to $11 \mathrm{~cm}$ (cf. Tables 5 with 7). This is another indication that GOCE/GRACE combined gravity field solutions (as is the case with GOCO01S) allows more accurate gravity field modelling than GOCE alone.

Table 5 Descriptive statistics of the differences EGM2008 minus GOCE-TIM (2...180) and EGM2008 minus GOCE-TIM (2...210) for various regions of Earth. The regions are the whole Earth, land, oceans (with $|\varphi|<90^{\circ}$ and $|\varphi|<83.3^{\circ}$, corresponding to GOCE's inclination) continents/land masses and selected areas Andes/Amazon Basin $\left(-20^{\circ} \leq \varphi \leq 10^{\circ},-80^{\circ} \leq \lambda \leq-50\right)$, Central Africa ($\left.20^{\circ} \leq \varphi \leq 10^{\circ}, 10^{\circ} \leq \lambda \leq 40^{\circ}\right)$ and Himalayas $\left(20^{\circ} \leq \varphi \leq 40^{\circ}, 65^{\circ} \leq \lambda \leq 100^{\circ}\right)$. NZ= New Zealand. The second column lists the percentage area covered by the region (whole of the Earth $=100 \%$ ). Unit of descriptive statistics is metres. 


\begin{tabular}{|c|c|c|c|c|c|c|c|c|c|}
\hline & & Min & Max & Mean & RMS & Min & Max & Mean & RMS \\
\hline Earth & 100 & -3.59 & 6.82 & 0.05 & 0.58 & -3.90 & 6.93 & 0.05 & 0.60 \\
\hline Land & 29.5 & -3.59 & 6.82 & 0.10 & 0.83 & -3.90 & 6.93 & 0.10 & 0.86 \\
\hline Ocean & 70.5 & -2.85 & 3.98 & 0.03 & 0.38 & -2.67 & 4.23 & 0.03 & 0.40 \\
\hline Earth $\left(|\varphi|<83.3^{\circ}\right)$ & 99.3 & -3.59 & 2.62 & 0.00 & 0.14 & -3.90 & 3.35 & 0.00 & 0.21 \\
\hline Land $\left(|\varphi|<83.3^{\circ}\right)$ & 29.1 & -3.59 & 2.62 & 0.00 & 0.20 & -3.90 & 3.35 & 0.00 & 0.28 \\
\hline Ocean $\left(|\varphi|<83.3^{\circ}\right)$ & 70.2 & -1.17 & 0.95 & 0.00 & 0.10 & -1.55 & 1.27 & 0.00 & 0.17 \\
\hline Australia & 1.5 & -0.32 & 0.27 & 0.01 & 0.08 & -0.58 & 0.61 & 0.00 & 0.15 \\
\hline NZ, Oceanian Islands $\left(\varphi<-12^{\circ}\right)$ & 0.1 & -0.44 & 0.34 & -0.03 & 0.14 & -0.91 & 0.68 & -0.01 & 0.25 \\
\hline South America & 3.6 & -2.03 & 2.62 & 0.00 & 0.34 & -2.55 & 3.35 & 0.00 & 0.40 \\
\hline North America & 4.4 & -0.65 & 0.61 & 0.00 & 0.08 & -0.80 & 0.77 & 0.00 & 0.14 \\
\hline Greenland & 0.4 & -0.39 & 0.37 & 0.00 & 0.12 & -0.65 & 0.63 & 0.01 & 0.16 \\
\hline Europe & 1.4 & -0.35 & 0.37 & 0.00 & 0.07 & -0.65 & 0.63 & 0.00 & 0.15 \\
\hline Africa & 5.9 & -1.58 & 2.06 & 0.00 & 0.23 & -1.89 & 2.35 & 0.00 & 0.28 \\
\hline Asia & 9.5 & -3.59 & 2.18 & 0.00 & 0.19 & -3.90 & 2.58 & 0.00 & 0.23 \\
\hline Antarctica $\left(|\varphi|<83.3^{\circ}\right)$ & 2.4 & -1.01 & 1.06 & -0.01 & 0.23 & -1.76 & 1.60 & -0.01 & 0.39 \\
\hline Andes/Amazon Basin & 2.2 & -2.03 & 2.62 & -0.01 & 0.40 & -2.55 & 3.35 & -0.01 & 0.46 \\
\hline Central Africa & 2.2 & -1.58 & 2.06 & 0.00 & 0.30 & -1.89 & 2.35 & 0.00 & 0.34 \\
\hline Himalayas & 1.5 & -3.59 & 2.18 & 0.00 & 0.42 & -3.90 & 2.58 & 0.00 & 0.46 \\
\hline
\end{tabular}

Table 6 Descriptive statistics of the differences EGM2008 minus ITG-GRACE2010s (2..160) and EGM2008 minus ITG-GRACE2010s (2..180) for various regions of Earth, unit is metres.

\begin{tabular}{|c|c|c|c|c|c|c|c|c|}
\hline \multirow[t]{2}{*}{ Area } & \multirow[t]{2}{*}{$\%$} & \multicolumn{4}{|c|}{$\begin{array}{c}\text { EGM2008-itg-grace2010s } \\
(2 . .160)\end{array}$} & \multicolumn{3}{|c|}{$\begin{array}{c}\text { EGM2008-itg-grace2010s } \\
(2 . .180)\end{array}$} \\
\hline & & Min & Max & Mean & RMS & Min & Max Mean & RMS \\
\hline Earth & 100 & -3.16 & 2.14 & 0.00 & 0.11 & -3.33 & 2.570 .00 & 0.26 \\
\hline Land & 29.5 & -3.16 & 2.14 & 0.00 & 0.15 & -3.33 & $2.57 \quad 0.00$ & 0.28 \\
\hline Ocean & 70.5 & -0.75 & 0.69 & 0.00 & 0.08 & -1.28 & 1.320 .00 & 0.24 \\
\hline Australia & 1.5 & -0.32 & 0.29 & 0.00 & 0.08 & -1.01 & $0.94 \quad 0.00$ & 0.29 \\
\hline NZ, Oceanian Islands $\left(\varphi<-12^{\circ}\right)$ & 0.1 & -0.22 & 0.21 & -0.01 & 0.09 & -0.59 & $0.50-0.01$ & 0.21 \\
\hline South America & 3.6 & -1.73 & 2.14 & 0.00 & 0.30 & -2.20 & $2.57 \quad 0.00$ & 0.41 \\
\hline North America & 4.4 & -0.49 & 0.37 & 0.00 & 0.07 & -0.88 & 0.780 .00 & 0.19 \\
\hline Greenland & 0.4 & -0.54 & 0.40 & 0.00 & 0.10 & -0.90 & 0.720 .00 & 0.20 \\
\hline Europe & 1.4 & -0.28 & 0.31 & 0.00 & 0.07 & -0.72 & 0.650 .00 & 0.21 \\
\hline Africa & 5.9 & -1.46 & 1.46 & 0.00 & 0.21 & -1.83 & 2.250 .00 & 0.31 \\
\hline Asia & 9.5 & -3.16 & 1.89 & 0.00 & 0.18 & -3.33 & $2.40 \quad 0.00$ & 0.28 \\
\hline Antarctica $\left(|\varphi|<83.3^{\circ}\right)$ & 2.4 & -0.54 & 0.57 & 0.00 & 0.10 & -1.25 & $1.76 \quad 0.00$ & 0.31 \\
\hline Andes/Amazon Basin & 2.2 & -1.73 & 2.14 & 0.00 & 0.35 & -2.20 & 2.570 .00 & 0.44 \\
\hline Central Africa & 2.2 & -1.46 & 1.46 & 0.00 & 0.27 & -1.83 & 2.250 .00 & 0.38 \\
\hline Himalayas & 1.5 & -3.16 & 1.89 & 0.00 & 0.39 & -3.33 & $2.40 \quad 0.00$ & 0.46 \\
\hline
\end{tabular}


Table 7 Descriptive statistics of the differences EGM2008 minus GOCO01S (2..180) and EGM2008 minus GOCO01S (2..210) for various regions of Earth, unit is metres.

\begin{tabular}{lccccccccc}
\hline \multirow{2}{*}{ Region } & \% & \multicolumn{3}{c}{ EGM2008-GOCO01S (2..180) } & \multicolumn{3}{c}{ EGM2008-GOCO01S (2..210) } \\
& & Min & Max & Mean & RMS & Min & Max & Mean & RMS \\
\hline Earth & 100 & -3.59 & 2.56 & 0.00 & 0.13 & -3.88 & 3.28 & 0.00 & 0.23 \\
Land & 29.5 & -3.59 & 2.56 & 0.00 & 0.19 & -3.88 & 3.28 & -0.01 & 0.32 \\
Ocean & 70.5 & -1.12 & 0.71 & 0.00 & 0.08 & -1.52 & 1.09 & 0.00 & 0.17 \\
\hline Earth $\left(|\varphi|<83.3^{\circ}\right)$ & 99.3 & -3.59 & 2.56 & 0.00 & 0.13 & -3.88 & 3.28 & 0.00 & 0.21 \\
Land $\left(|\varphi|<83.3^{\circ}\right)$ & 29.1 & -3.59 & 2.56 & 0.00 & 0.19 & -3.88 & 3.28 & 0.00 & 0.28 \\
Ocean $\left(|\varphi|<83.3^{\circ}\right)$ & 70.2 & -1.12 & 0.71 & 0.00 & 0.08 & -1.52 & 1.09 & 0.00 & 0.16 \\
\hline Australia & 1.5 & -0.28 & 0.26 & 0.00 & 0.07 & -0.52 & 0.61 & 0.00 & 0.15 \\
NZ, Oceanian Islands $\left(\varphi<-12^{\circ}\right)$ & 0.1 & -0.42 & 0.25 & -0.02 & 0.12 & -0.90 & 0.69 & 0.00 & 0.24 \\
South America & 3.6 & -2.04 & 2.56 & 0.00 & 0.33 & -2.55 & 3.28 & 0.00 & 0.39 \\
North America & 4.4 & -0.68 & 0.52 & 0.00 & 0.07 & -0.77 & 0.72 & 0.00 & 0.14 \\
Greenland & 0.4 & -0.45 & 0.36 & 0.00 & 0.11 & -0.69 & 0.58 & 0.00 & 0.16 \\
Europe & 1.4 & -0.33 & 0.37 & 0.00 & 0.07 & -0.63 & 0.61 & 0.00 & 0.14 \\
Africa & 5.9 & -1.51 & 2.03 & 0.00 & 0.23 & -1.87 & 2.33 & 0.00 & 0.27 \\
Asia & 9.5 & -3.59 & 2.19 & 0.00 & 0.18 & -3.88 & 2.62 & 0.00 & 0.23 \\
Antarctica $\left(|\varphi|<83.3^{\circ}\right)$ & 2.4 & -0.93 & 1.00 & 0.00 & 0.22 & -1.74 & 1.60 & 0.00 & 0.39 \\
\hline Andes/Amazon Basin & 2.2 & -2.04 & 2.56 & 0.00 & 0.39 & -2.55 & 3.28 & 0.00 & 0.45 \\
Central Africa & 2.2 & -1.51 & 2.03 & 0.00 & 0.30 & -1.87 & 2.33 & 0.00 & 0.34 \\
Himalayas & 1.5 & -3.59 & 2.19 & 0.00 & 0.42 & -3.88 & 2.62 & 0.00 & 0.46 \\
\hline
\end{tabular}


EGM2008 - GOCE-TIM band 2 .. 180
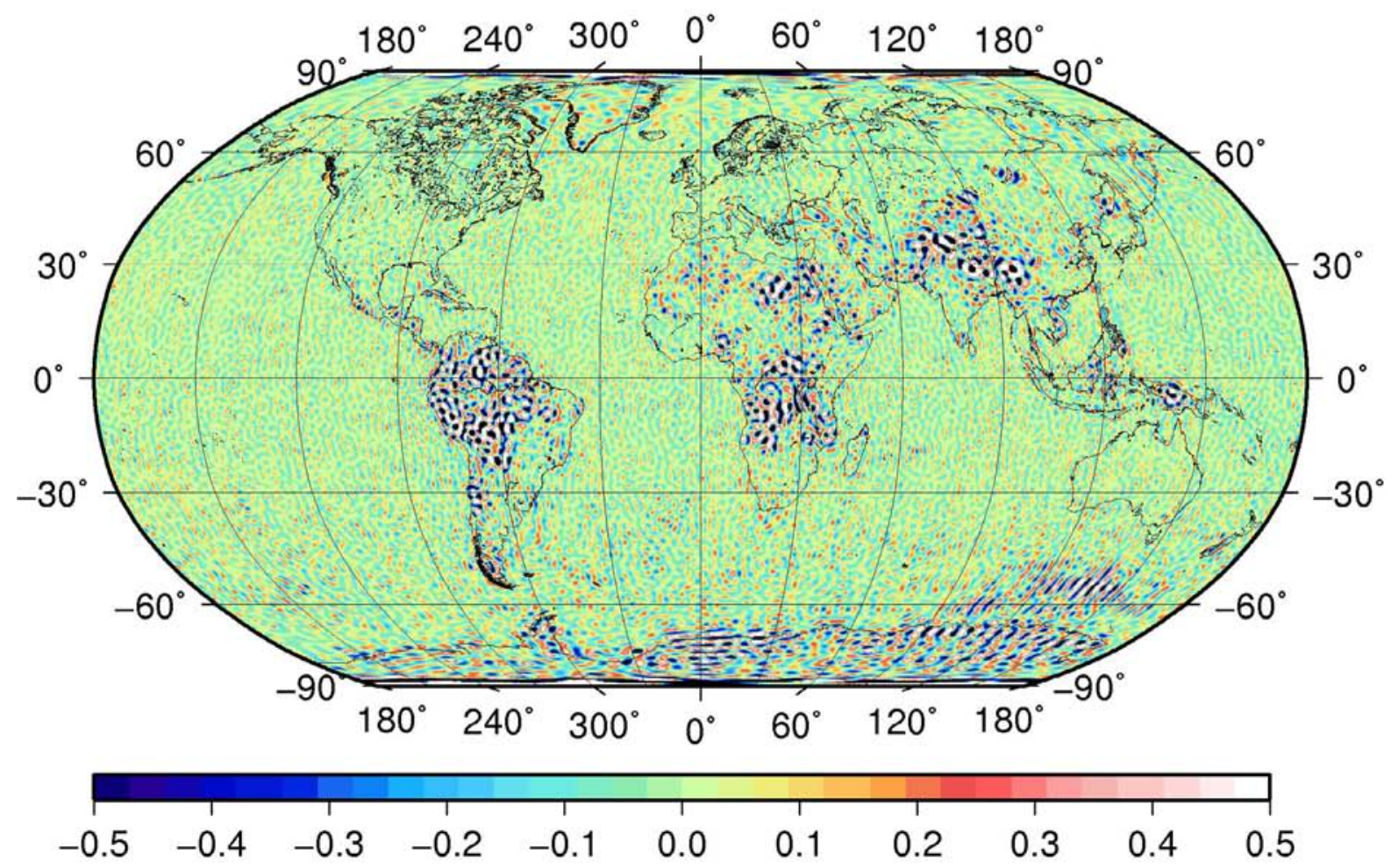

EGM2008 - GOCE-TIM band 2 .. 210
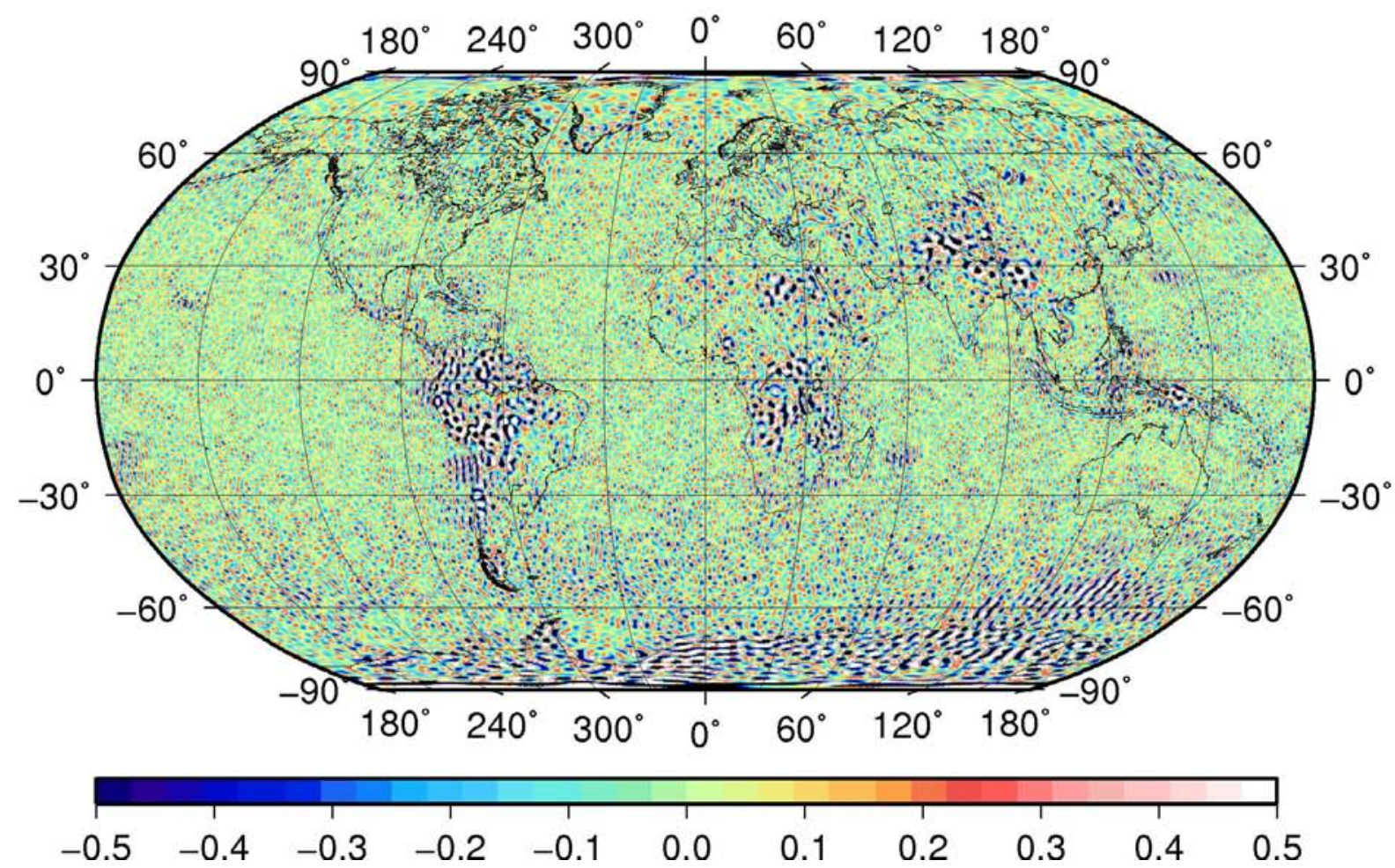

Fig.11 Top: EGM2008 minus GOCE-TIM quasigeoid heights in spectral band 2 to 180 . Bottom: EGM2008 minus GOCE-TIM quasigeoid heights in spectral band 2 to 210. Units are metres 
EGM2008 - itg-grace2010s band 2 .. 160
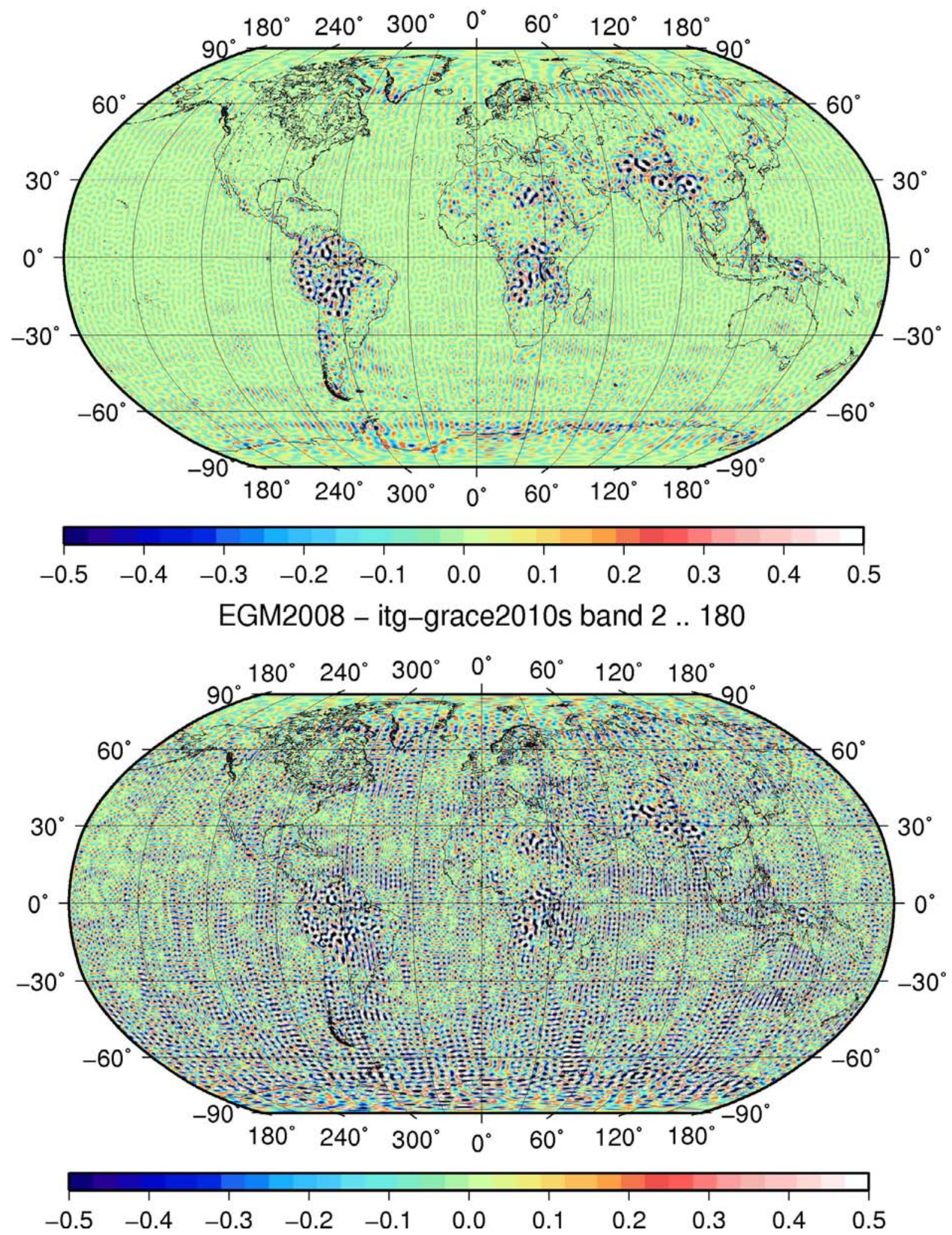

Fig.12 Top: EGM2008 minus ITG-GRACE2010S quasigeoid heights in spectral band 2 to 160. Bottom: EGM2008 minus ITG-GRACE2010S quasigeoid heights in spectral band 2 to 180. Units are metres 

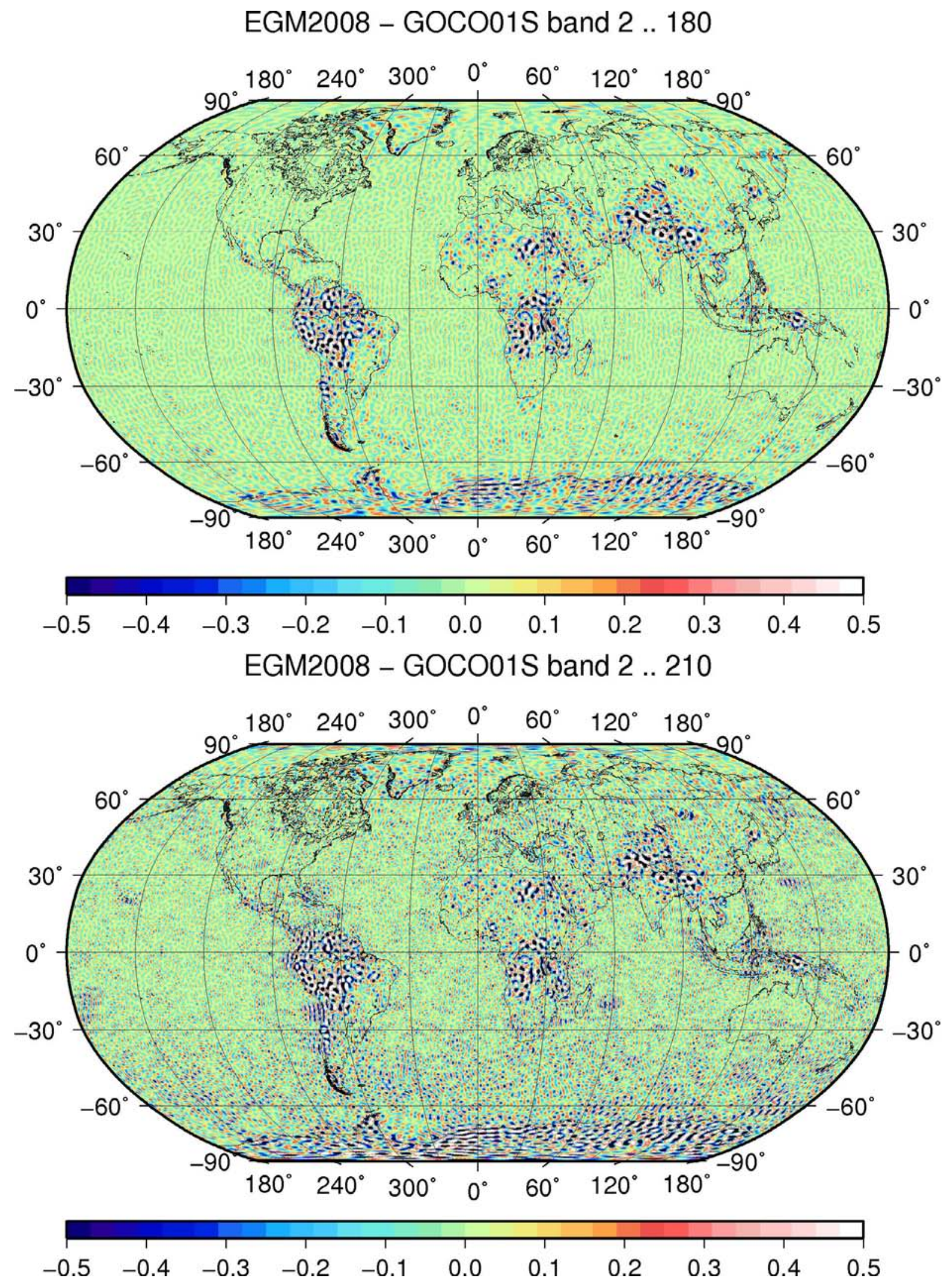

Fig.13 Top: EGM2008 minus GOCO01S quasigeoid heights in spectral band 2 to 180 . Bottom: EGM2008 minus GOCO01S quasigeoid heights in spectral band 2 to 210. Units are metres 


\section{Conclusions}

We have evaluated the first four GOCE-based GGMs in comparison to two GRACE-only GGMs and EGM2008. As a first assessment method, we used terrestrial gravity and vertical deflection data sets (Sects. 2 to 4). To overcome the problem of different spectral content, we applied a spectral enhancement method (SEM), where EGM2008 and RTM data are used to better match the spectral bands. Adding omission error estimates from EGM2008 and RTM data greatly reduces the discrepancies between the GGMs and terrestrial observations. As a second assessment method, we compared global grids of EGM2008 and GOCEbased/GRACE-based quasigeoid heights in different spectral bands (Sect. 5). This is a simple and powerful evaluation technique for global GOCE evaluation. The method circumvents the problem of different spectral content and benefits from the high-quality of EGM2008 over well-surveyed areas.

The terrestrial gravity and vertical deflection data sets turned out to be very sensitive to indicate the degree beyond which signal loss occurs; however, direct insight into the GOCE-performance is gained only over the data areas and not outside. Complementarily to this, the global EGM2008-based quasigeoid comparisons give world-wide insight into the GOCE-performance. Because of the different data coverage (regional vs. global), gravity field functionals (gravity, deflections and quasigeoid) and different handling of signal omission (modelling vs. avoidance) the results from both assessment strategies are used together to evaluate the first GOCE gravity field models.

As the main outcome of this study, any of the evaluated GOCE-based GGMs seem to advance the knowledge of Earth's global gravity field in comparison to ITG-GRACE2010S as a benchmark of the 'pre-GOCE-era'. Both the vertical deflections and the terrestrial gravity measurements 'sensed' improvements in the spectral band $\sim 160-165$ to $180-185$. The spectral band was confirmed by comparisons involving global grids of EGM2008 quasigeoid heights. Given that spherical harmonic degrees 180 to 185 are equivalent to a spatial resolution of $\sim 107$ to $\sim 110 \mathrm{~km}$, after two months, GOCE is already near the targeted resolution of $\sim 100 \mathrm{~km}$. The prospects for the complete mission are therefore good.

The comparisons against ground-truth vertical deflections and gravity anomalies showed GOCO01S, GOCE-TIM and GOCE-SPW to experience signal loss near degrees $\sim 180-185$, which is below their nominal model resolution (spherical harmonic degree 210 and 224, respectively). This suggests that the models are somewhat underpowered in their high frequencies, which is expected from the decay of the gravity field with altitude. Given that the evaluated first GOCE-GGMs are based on only two months of data, improvements may be possible for future models based on more observations.

The GOCE-based models showed - up to their degree of signal loss - a performance fairly equivalent to EGM2008 over areas with very good pre-GOCE gravity field knowledge. This observation was made not only from the comparisons using ground-truth vertical deflections and terrestrial gravity (Sect. 4), but consistently also from EGM2008 quasigeoid heights over Australia, Europe, North America and the oceans, where EGM2008's quality is high (Sect 5). Complementarily, we found large discrepancies between EGM2008 and GOCE quasigeoid heights over parts of South America, Africa, Asia and Antarctica, which are the EGM2008 'problem' areas (Sect. 5). Holistically, these observations provide strong evidence 
that the two months of GOCE observations improve the gravity field knowledge of our planet in the spherical harmonic window of degrees $~ 160-165$ to $~ 180-185$.

\section{Acknowledgements}

Christian Hirt is supported under the ARC's Discovery Projects funding scheme (project number DP0663020). Will Featherstone was the recipient of an Australian Research Council (ARC) Professorial Fellowship (project number DP0663020). The views expressed herein are those of the authors and are not necessarily those of the ARC. We are very grateful to the suppliers of the data sets utilised in our study. We thank Beat Bürki (ETH Zürich), Urs Marti (swisstopo), Swiss Geodetic Commission, Geoscience Australia and Landgate. Naturally, the producers of the new GOCE models are thanked for making the new GOCE models so readily available via ESA and ICGEM. Parts of the figures were produced with the Generic Mapping Tools (Wessel and Smith 1998). Thanks are extended to the reviewers and editors for comments on the manuscript.

\section{References}

Andersen OA, Knudsen P, Berry PAM (2010) The DNSC08GRA global marine gravity field from double retracked satellite altimetry. J Geod. 84(3), 191-199, DOI 10.1007/s00190009-0355-9.

Bürki B, Somieski, AE, Sorber P, Kahle H-G, Hirt C (2007) The digital astronomical deflection measuring system DIADEM. Swiss National Rep. on the Geodetic Activities in the Years 2003-2007, Presented to the XXIV General Assembly of the IUGG in Perugia, Italy, Swiss Geodetic Commission, pp 143-144

Bruinsma SL, Marty JC, Balmino G, Biancale R, Förste C, Abrikosov O, Neumayer H (2010) GOCE Gravity Field Recovery by Means of the Direct Numerical Method. Presented at the ESA Living Planet Symposium 2010, Bergen, June 27 - July 2 Bergen, Norway

Drinkwater, M.R., R. Floberghagen, R. Haagmans, D. Muzi, and A. Popescu (2003). GOCE: ESA's first Earth Explorer Core mission. In Beutler, G.B., M. R. Drinkwater, R. Rummel, and R. von Steiger, Earth Gravity Field from Space - from Sensors to Earth Sciences. In the Space Sciences Series of ISSI, Vol. 18, 419-432, Kluwer Academic Publishers, Dordrecht, Netherlands ISBN: 1-4020-1408-2/

EGM Development Team (2009) Description of files Containing Propagated Error Estimates From EGM2008 on Global 5' × 5' Grids, U.S. National Geospatial Intelligence Agency (NGA). Available at: http://earth-info.nga.mil/GandG/wgs84/gravitymod/

Featherstone, WE (2007) Augmentation of AUSGeoid98 with GRACE satellite gravity data, J Spatial Sci 52(2): 75-86

Featherstone WE, Morgan L (2007) Validation of the AUSGeoid98 model in Western Australia using historic astrogeodetically observed deviations of the vertical. J Royal Soc West Aust 90(3): 143-149

Featherstone WE, Lichti DD (2009) Fitting gravimetric geoid models to vertical deflections. J Geod 83(6): 583-589. doi: 10.1007/s00190-008-0263-4 
Featherstone WE, Kirby JF, Hirt C, Filmer MS, Claessens SJ, Brown NJ, Hu H, Johnston GM (2011) The AUSGeoid09 model of the Australian Height Datum. J Geod, 85(3): 133150. doi: 10.1007/s00190-010-0422-2

Forsberg R, Tscherning CC (1981) The Use of Height Data in Gravity Field Approximation by Collocation. Journal of Geophysical Research 86 No B9: 7843-7854

Forsberg R (1984) A study of terrain reductions, density anomalies and geophysical inversion methods in gravity field modelling. Report 355, Department of Geodetic Science and Surveying, Ohio State University, Columbus

GOCO Consortium (2010) The satellite-only global gravity field model GOCO01S derived from GOCE and GRACE. http://inas.tugraz.at/GOCO/ and http://icgem.gfzpotsdam.de/ICGEM/ICGEM.html

Gruber T (2004) Validation concepts for gravity field models from satellite missions. in: Lacoste, H. (eds.) Proceedings of the 2nd International GOCE User Workshop "GOCE, The Geoid and Oceanography”, ESA SP-569, ESA

Gruber T (2008) The GOCE gravity field space mission as an important step for the exploration of our planet. Proceedings of INCOSE2008 - Systems Engineering for the Planet, International Council of System Engineering (INCOSE), 14 pages

Gruber T (2009) Evaluation of the EGM2008 gravity field by means of GPS-levelling and sea surface topography solutions. External quality evaluation reports of EGM08, Newton's Bulletin 4:3-17, Bureau Gravimétrique International (BGI) / International Geoid Service (IGeS)

Gruber T, Visser PNAM, Ackermann C, Hosse M (2011) Validation of GOCE gravity field models by means of orbit residuals and geoid comparisons. J Geod, submitted

Hirt C, Flury J (2008) Astronomical-topographic levelling using high-precision astrogeodetic vertical deflections and digital terrain model data. J Geod 82(4-5): 231-248. DOI:10.1007/s00190-007-0173

Hirt C, Seeber G (2008) Accuracy Analysis of vertical deflection data observed with the Hannover Digital Zenith Camera System TZK2-D. J Geod 82(6): 347-356. DOI: 10.1007/s00190-007-0184-7

Hirt C (2010) Prediction of vertical deflections from high-degree spherical harmonic synthesis and residual terrain model data. J Geod 84(3):179-190. DOI 10.1007/s00190-009-0354$\mathrm{x}$

Hirt C, Marti U, Bürki B, Featherstone WE (2010a) Assessment of EGM2008 in Europe using accurate astrogeodetic vertical deflections and omission error estimates from SRTM/DTM2006.0 residual terrain model data. J Geophys Res Solid Earth 115, B10404, DOI:10.1029/2009JB007057

Hirt C, Featherstone WE, Marti U (2010b) Combining EGM2008 and SRTM/DTM2006.0 residual terrain model data to improve quasigeoid computations in mountainous areas devoid of gravity data. J Geod 84(9): 557-567. DOI 10.1007/s00190-010-0395-1 
Hirt C, Bürki B, Somieski A, Seeber G (2010c) Modern determination of vertical deflections using digital zenith cameras. J Surv Eng 136(1), 1-12. DOI: 10.1061/_ASCE_SU.19435428.0000009

Holmes, SA, Pavlis NK (2008) Spherical harmonic synthesis software harmonic_synth. http://earth-info.nga.mil/GandG/wgs84/gravitymod/new_egm/new_egm.html

Ihde, J, Wilmes, H, Müller J, Denker, H, Voigt C, Hosse M (2010) Validation of Satellite Gravity Field Models by Regional Terrestrial Data sets. In: System Earth via GeodeticGeophysical Space Techniques Advanced Technologies in Earth Sciences, Part 3, pp 277-296

Jarvis A, Reuter HI, Nelson A, and Guevara E (2008) Hole-filled SRTM for the globe Version 4, Available from the CGIAR-SXI SRTM 90m database: http://srtm.csi.cgiar.org

Jekeli C (1999) An analysis of vertical deflections derived from high-degree spherical harmonic models, J Geod 73(1), 10-22, doi: 10.1007/s001900050213

Kaula WM (1966) Theory of Satellite Geodesy. Blaisdel, Waltham

Marti U (2004) High-precision combined geoid determination in Switzerland. Presented at Gravity, Geoid and Space Missions (GGSM) 2004 Symposium, Porto, Aug 30 - Sep 03 2004, Porto, Portugal

Mayer-Gürr T (2007) ITG-Grace03s: The latest GRACE gravity field solution computed in Bonn. Joint Int. GSTM and SPP Symposium, 15-17 Oct. 2007, Potsdam, Germany, http://www.geod.uni-bonn.de/itg-grace03.html

Mayer-Gürr T, Kurtenbach E, Eicker A (2010) ITG-Grace2010 Gravity Field Model. http://www.igg.uni-bonn.de/apmg/index.php?id=itg-grace2010, 2010

Metzler B, Pail R (2005) GOCE data processing: the Spherical Cap Regularization Approach. Stud Geophys Geod, 49:441-462. doi:10.1007/s11200-005-0021-5

Migliaccio F, Reguzzoni M, Sanso F, Tscherning CC, Veicherts M (2010) GOCE data analysis: the space-wise approach and the first space-wise gravity field model. Presented at the ESA Living Planet Symposium 2010, Bergen, June 27 - July 2, Bergen, Norway

Murray AS (1997) The Australian national gravity database, AGSO Journal of Australian Geology \& Geophysics 17: 145-155

Nagy D, Papp G, and Benedek J (2000) The gravitational potential and its derivatives for the prism J. Geod 74(7-8):552-560. DOI: 10.1007/s001900000116. Erratum in J. Geod. 76(8):475. DOI: 10.1007/s00190-002-0264-7

Newton's Bulletin (2009) External quality evaluation reports of EGM08. Issue 4, Bureau Gravimétrique International (BGI) / International Geoid Service (IGeS)

Pail R, Goiginger H, Schuh W-D, Höck E, Brockmann JM, Fecher T, Gruber T, Mayer-Gürr T, Kusche J, Jäggi A, Rieser D (2010a) Combined satellite gravity field model GOCO01S derived from GOCE and GRACE. Geophys Res Lett 37, L20314, DOI: 10.1029/2010GL044906 
Pail R, Goiginger H, Mayrhofer R, Schuh W-D, Brockmann JM, Krasbutter I, Höck E, Fecher $\mathrm{T}$ (2010b), Global gravity field model derived from orbit and gradiometry data applying the time-wise method. Presented at the ESA Living Planet Symposium 2010, Bergen, June 27 - July 2, Bergen, Norway

Pail R, Bruinsma S, Migliaccio F, Förste C, Goiginger H, Schuh W-D, Höck E, Reguzzoni M, Brockmann JM, Abrikosov O, Veicherts M, Fecher T, Mayrhofer R, Krasbutter I, Sansò F, Tscherning CC (2011). First GOCE gravity field models derived by three different approaches. Journal of Geodesy, submitted

Pavlis NK, Saleh J (2004) Error Propagation with Geographic Specifity for Very High Degree Geopotential Models, Proceed. GGSM 2004 IAG International Symposium Porto, Portugal (ed. C. Jekeli et al.), Springer, Heidelberg: 149-154

Pavlis NK, Factor JK, Holmes SA (2007) Terrain-related gravimetric quantities computed for the next EGM. Proceedings of the 1st International Symposium of the International Gravity Field Service (IGFS), Istanbul, pp 318-323

Pavlis NK, Holmes SA, Kenyon SC, Factor JK (2008) An Earth Gravitational Model to Degree 2160: EGM2008. Presented at the 2008 General Assembly of the European Geoscience Union, Vienna, Austria, April 13-18, 2008

Reigber C, Balmino G, Schwintzer P, Biancale R, Bode A, Lemoine J-M, König R, Loyer S, Neumayer H, Marty J-C, Barthelmes F, Perosanz F, Zhu SY (2002) A high quality global gravity field model from CHAMP GPS tracking data and accelerometry (EIGEN-1S). Geophys Res Lett, 29(14), 10.1029/2002GL015064, 2002

Reigber C, Schmidt R, Flechtner F, König R, Meyer U, Neumayer K-H, Schwintzer P, Zhu SY (2005) An Earth gravity field model complete to degree and order 150 from GRACE: EIGEN-GRACE02S. J Geodyn 39(1):1-10

Rummel R, Balmino G, Johannessen J, Visser P, Woodworth P (2002) Dedicated gravity field missions - principles and aims. J Geodyn 33(1-2):3-20

Rummel R, Gruber T, Koop R (2004) High Level Processing Facility for GOCE: products and processing strategy. in: Lacoste, H. (eds.) Proceedings of the 2nd International GOCE User Workshop “GOCE, The Geoid and Oceanography”, ESA SP-569, 2004

Rummel R (2005) Geoid and gravity in earth sciences - an overview. Workshop on Enabling Observation Techniques for Future Solid Earth Missions, Jan 30-Feb 01, 2003, Int Space Sci Inst Bern Switzerland, Earth Moon and Planets 94(1-2):3-11

Rummel R, Gruber T, Flury J, Schlicht A (2009) ESA's Gravity Field and Steady-State Ocean Circulation Explorer GOCE. Zeitschrift für Vermessungswesen 3/2009:125-130

Schwarz K-P (1985) Data types and their spectral properties. UCSE Report Nr. 60003. Division of Surveying Engineering, University of Calgary, Calgary, Alberta, Canada

Tapley B, Ries J, Bettadpur S, Chambers D, Cheng M, Condi F, Gunter B, Kang Z, Nagel P, Pastor R, Pekker T, Poole S, Wang F (2005) GGM02 - An improved Earth gravity field model from GRACE. J Geod 79(8): 467-478. DOI: 10.1007/s00190-005-0480-z 
Tapley B, Ries J, Bettadpur S, Chambers D, Cheng M, Condi F, Poole S (2007) The GGM03 Mean Earth Gravity Model from GRACE. Eos Trans. AGU 88(52), Fall Meeting of American Geophysical Union 2007, Abstract G42A-03

Torge W (2001) Geodesy. 3rd Edition, de Gruyter, Berlin, New York

Visser PNAM, Rummel R, Balmino G, Sünkel H, Johannessen J, Aguirre M, Woodworth PL, Le Provest C, Tscherning CC, Sabadini R (2002) The European Earth Explorer Mission GOCE: Impact for the geosciences. Ice Sheets, Sea Level and the Dynamic Earth 29: 95-107

Voigt C, Denker H, Hirt C (2009) Regional astrogeodetic validation of GPS/levelling data and quasigeoid models. in: Sideris M.G (ed) Observing Our Changing Earth, Springer, Berlin Heidelberg New York, pp 413-420

Wessel P, Smith WHF (1998) New, improved version of the Generic Mapping Tools released. EOS Trans. AGU 79:579 